\title{
Experimental Study of the Structure of a Wingtip Vortex
}

\author{
Dr. Elgin A. Anderson \\ Christopher T. Wright \\ Mechanical and Aerospace Engineering Department \\ Utah State University \\ Logan, UT 84322-4130
}

February 2000

Summary of Research for period February 1998 to December 1999

Grant Number NAG-1-1999

\author{
Dr. Thomas Gatski \\ NASA Langley Research Center \\ Hampton, VA 23681
}




\section{SUMMARY}

A complete look at the near-field development and subsequent role-up of a wingtip vortex from a NACA 0015 wing section is investigated. Two separate but equally important surveys of the vortex structure in the region adjacent to the wingtip and approximately one chord length downstream of the trailing edge are performed. The two surveys provide qualitative flow visualization and quantitative velocity measurement data. The near-field development and subsequent role-up of the vortex structures is strongly influenced by the angle-of-attack and the end-cap treatment of the wing section. The velocity field near the wingtip of the NACA 0015 wing section was measured with a triple-sensor hot wire probe and compared to flow visualization images produced with titanium tetrachloride smoke injection and laser illumination. The flat end-cap results indicate the formation of multiple, relatively strong vortex structures as opposed to the formation of a single vortex produced with the round end-cap. The multiple vortices generated by the flat end-cap are seen to rotate around a common center in a helical pattern until they eventually merge into a single vortex. Compared to a non-dimensional loading parameter, the results of the velocity and flow visualization data shows a "jetlike" axial velocity profile for loading parameter values on the order of 0.1 and a "wakelike" profile for much lower loading parameter values. 
CONTENTS

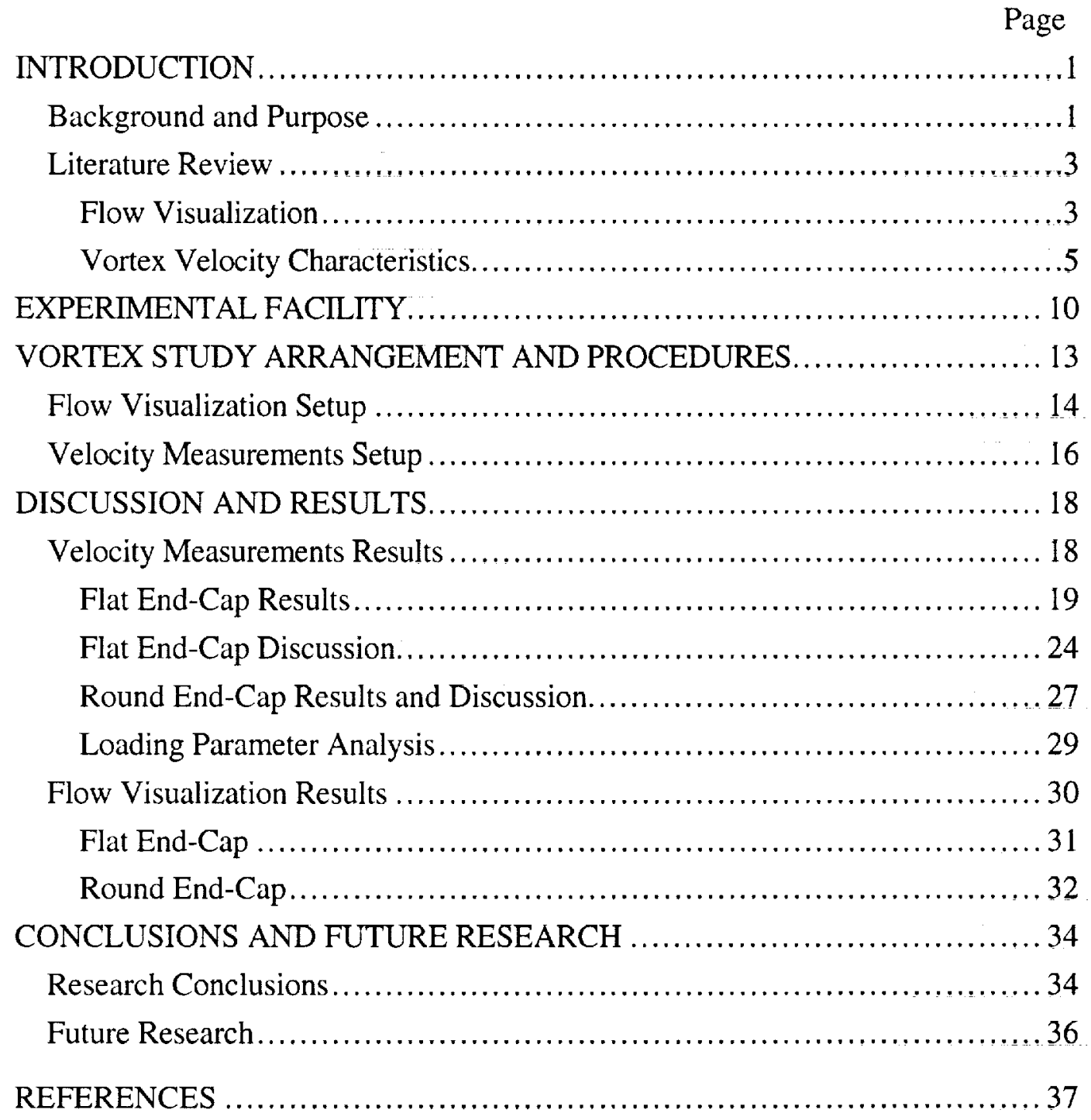




\section{CHAPTER 1}

\section{INTRODUCTION}

\section{Background and Purpose}

The development of a wingtip vortex occurs any time a lifting surface, or wing, terminates in a fluid. The formation of this vortex can be understood if one considers the pressure field that exists near the wingtip. As a wing moves through a fluid, lift is generated when a low pressure field is hydrodynamically produced on the top surface or suction side of the wing and a relatively higher pressure field is produced on the bottom surface or pressure side of the wing. This large pressure difference between the two sides of the wing causes the fluid to accelerate around the tip of the wing from pressure side to suction side, thus forming a vortex as shown in Figure 1. This vortex trails from the wingtip and remains relatively strong for many chord lengths downstream.

The characteristics, which determine the behavior of wingtip vortices, have been the subject of numerous experimental and numerical studies. The important effects vortices have on practical problems have primarily driven the popularity of such studies. Reports by Arndt (1991), Rossow et. al (1995), and Sarpkaya (1998) suggest that these problems consist of the separation distances between aircraft in high lift configurations such as takeoff and landing, the interactions between shed vortices and following helicopter rotor blades, and the vibrational noise caused by submarine sails and propeller cavitation on ships and aircraft. A general goal of these studies is to reduce the inefficient or hazardous effects trailing vortices have on following lifting surfaces. Despite the numerous studies performed to date there is much left to be determined regarding the structure of the vortices. A more detailed understanding of the vortex structure and the mechanisms, which determine its development and behavior within a few chord lengths behind the lifting body, will help make progress towards achieving this goal. 
The main purpose of this project is to investigate the characteristics of a wingtip vortex generated from a NACA 0015 rectangular wing section. Of primary interest are the mechanisms and characteristics of the flow field, which govern the initial development, the roll-up, and the velocity distributions of the generated vortex. These characteristics are studied qualitatively with smoke visualization techniques and quantitatively with hot wire probe velocity measurements. The smoke visualization experiments provide video footage and still images of the development and initial roll-up of the primary and secondary shear layer vortices. This visual survey begins at the leading edge of the wing section and ends one chord length downstream of the wing's trailing edge. The velocity measurements provide both tangential and axial velocity profiles within the trailing vortex. The tangential and axial velocity profiles are used to determine the size and strength, and the magnitude of the peak velocity, respectively, of the vortex core. These velocity profiles are obtained at several locations between the trailing edge and one chord length downstream of the wing section. Both the visual and velocity experiments are conducted at a specific Reynolds number and three different angles-of-attack with the use of a flat and rounded end-cap.

A secondary purpose of this project is to provide accurate and detailed information on the characteristics that govern vortex flow fields, which can be extended to the behavior of a vortex generated from a real airplane flying in the atmosphere. Because the unbounded conditions that apply to a vortex generated in the atmosphere are more easily simulated in the unbounded domain of a computer model than in a wind tunnel, the information collected in this study could be used to validate computational algorithms. This will be accomplished by adding to the current database of experimental vortex characteristics. 
Literature Review

A review of published papers on the subject of wingtip vortices reveals numerous experimental and numerical data on several specific areas of vortex development, roll-up, structure, and behavior. Two of these areas in particular warrant further investigation due to their incomplete or conflicting results, and are of primary interest to this research. These two areas include a qualitative visual survey of the vortex development and role-up and a quantitative survey of the vortex velocity profiles. The next two sections present a brief review of the published papers, which discuss these two areas of interest.

\section{Flow Visualization}

A very useful tool in understanding the formation and role-up of a wingtip vortex is flow visualization. This technique is performed by introducing small neutrally buoyant particles or "smoke" upstream of the wing section; a process known as seeding the flow. The introduced smoke is entrained into the shear layers that form on the pressure side of the wing section and eventually into the forming vortex structures. Passing a laser sheet through the entrained smoke illuminates the small smoke particles and allows the flow to be visualized. A video camera is then used to capture video footage and still images of the 2-D vortex structures.

Experimental data from vortex visualization techniques, however, is sparse and covers only a small range of low Reynolds numbers. The lack of data is evidence of the difficulties encountered by experimentalists to first, adequately seed the flow, and second, to record the images with enough resolution to see the fine details of the vortex structures. Even with these difficulties, previous studies have shown significant differences in the development process for wing sections with flat end-caps vs. those with rounded endcaps. In general, these differences are the formation of multiple vortices around a flat endcap and a single vortex around a rounded end-cap. 
Using both hot wire probe and flow visualization techniques, Francis and Kennedy (1979) performed a study on the development of a vortex over a rectangular NACA 64009 wing section with a flat end-cap. Their results show that for a Reynolds number of 247,000 and an angle-of-attack of 4 degrees a single vortex formed at the abrupt edge of the flat end-cap on the pressure side of the wing section. This vortex entrained more fluid as it traveled along the edge of the flat end-cap until it eventually merged with a second vortex, which formed on the suction side of the wing section, near the trailing edge.

Later flow visualization studies performed by Francis and Katz (1988), and Katz and Galdo (1989) using a rectangular NACA-66 hydrofoil and Shekarriz et. al (1992, 1993) using a rectangular DARPA sail confirmed the formation of multiple vortex structures. Their studies, which were performed at Reynolds numbers between 37,000 and 380,000, and angles-of-attack greater than 4 degrees, showed the formation of multiple secondary vortex structures. The formation of these secondary vortices occurs in the region where the abrupt edges of the end-cap geometry cause the fluid to separate as it moves from the pressure side to the suction side of the wing section. In this separation region, the secondary vortices originate in the pressure side boundary layer and tend to be quite small. The difficulty in visualizing these secondary vortex structures then becomes the troublesome task of introducing the smoke particles into the boundary layer where they can be illuminated by the laser sheet.

A comparable flow visualization study performed by Liang and Ramaprian (1991) looked at the vortex development over a rectangular NACA 0015 wing section with a rounded end-cap. Unlike the wing sections with flat end-caps, their results showed the formation of a suction side vortex with no evidence of multiple vortices forming on the pressure side of the wing section. This should be expected since the geometry of a rounded end-cap dictates separation due to an adverse pressure gradient on the suction side of the wing section and not due to the abrupt edges on a flat end-cap. Their study 
was also conducted at relatively low Reynolds numbers $(80,000<\operatorname{Re}<194,000)$ and angles-of-attack between 5 and 15 degrees.

In addition to experimental studies, several numerical calculations have been performed to investigate the near-field development and structure of wing tip vortices. Based on the complexity of the problem, only wing sections with rounded or beveled endcap geometries have been investigated. The most resent studies, conducted by DaclesMariani et. al (1995) and Hsiao and Pauley (1996), simulated flow over rectangular untwisted NACA 0012 and 0015 wing sections, respectively. Though their results were calculated for much higher Reynolds numbers compared to those from similar experimental studies (1.5 and 3.5 million, and 4.6 million, respectively) they show the formation of only the primary suction side vortex. These results suggest that the formation of a wingtip vortex is strongly dependent on the end-cap treatment.

\section{Vortex Velocity Characteristics}

The foundation of our current understanding of wingtip vortex behavior stems predominately from experimentally collected velocity data. These measurements are made at discrete locations within the vortex flow field with the use of such tools as hot wire anemometers, particle image velocimeters (PIV), laser doppler velocimeters (LDV), and pressure probes. The vortex is typically described by two velocity components $U_{x}$ and $\mathrm{U}_{\theta}$, where $\mathrm{x}$ and $\theta$ are the axial (streamwise) and tangential directions respectively. Plots of the variation of these velocity components at a specific downstream location of the wingtip provide the basis of comparison between different experimental and numerical studies.

Due to widely varying experimental conditions, significant differences in the axial and tangential velocity profiles make it difficult to define what might be called a "typical" wingtip vortex. The axial velocity profile seems to be the main source of inconsistencies between studies (Ramaprian and Zheng 1997). In an attempt to analytically define a 
typical wingtip vortex, Batchelor (1964) looked at the characteristic features of a trailing line vortex. His analysis explained, in part, the relationship between the axial and tangential velocities based on a pressure gradient in each of these directions. This relationship is founded in the principles of Bernoulli, which are derived from the momentum equations of the flow field. In general, he concluded that due to the relationship between the pressure and velocity fields in a vortex a change in the tangential velocity can induces a proportional change in the axial velocity producing both an axial velocity acceleration and deceleration with respect to the free stream velocity. This effect on the characteristics of the vortex structure he terms the "Bernoulli effect." However, at the time of his study very little experimental data existed to support his analysis.

$\mathrm{n}$ the years following Batchelor's (1964) analytical study, several experimental studies were performed in which the axial velocity characteristics of the wingtip vortex were noted (Thompson 1975). Each of these studies, performed with widely differing test parameters and wing section configurations, presented results that showed both "jetlike" and "wake-like" axial velocity profiles. Unfortunately, the significance of Batchelor's (1964) study went unnoticed and the results from the subsequent experimental studies seemed to contradict each other when, in fact, they could be reconciled. Nonetheless, the results from these studies were different, and the likely reason was the effects varying test parameters and wing section configurations had on the vortex structure. However, test parameters and wing configurations from different studies rarely overlap making it difficult to determining which parameters and configurations influence the production of a "jet-like" or "wake-like" axial velocity" profile.

Comparisons of two recent experimental studies performed by Chow et. al (1997) and Devenport et. al (1996) provide an example of how varying test parameters and wing configurations can affect the structure of the vortex velocity flow field. The study performed by Chow et al. (1997) used a NACA 0012 rectangular wing section with a 
rounded end-cap and an aspect ratio of 0.75 . The wing section was positioned at 10 degrees angle-of-attack while measurements were taken at approximately 0.16 to 0.67 chord lengths downstream. Data taken from each location produced "jet-like" velocity profiles where the magnitude of the maximum axial velocity was approximately $1.7 \mathrm{U}_{\infty}$ decaying only slightly from the trailing edge to 0.67 chords. Devenport et al. (1996) performed a similar study using a NACA 0012 rectangular wing section with a flat endcap and an aspect ratio of 4.33. Their wing was positioned at 5 degrees angle-of-attack and velocity measurements were taken at various locations between 5 and 30 chord lengths behind the wing's trailing edge. Their results show that the axial velocity data produced a "wake-like" profile where the maximum core velocity was less than the free stream velocity with a magnitude of approximately $0.84 \mathrm{U}_{\infty}$. In addition, the magnitude of this velocity deficit changed very little from 5 to 30 chord lengths downstream.

It is likely that the different velocity profiles produced by the studies of Chow et al. (1997) and Devenport et al. (1996) are due to the differences in their test parameters. These differences are evident in the Reynolds number $\left(4.6 \times 10^{6}\right.$ vs. $\left.5.3 \times 10^{5}\right)$, the aspect ratio ( 0.75 vs. 4.33 ), the angle of attack ( 10 degrees vs. 5 degrees), and the test location in chord lengths downstream of the wingtip ( $\mathrm{x} / \mathrm{c}<0.67$ vs. $\mathrm{x} / \mathrm{c}>5.0$ ) for Chow et al. (1997) and Devenport et al. (1996) respectively. Furthermore, their data acquisition methods were different in that Chow et al. (1997) used a 7-hole pressure probe and Devenport et al. (1996) used a 4-sensor hot wire probe. Which of these parameters, or perhaps which combinations of these parameters, produce a "jet-like" vs. "wake-like" profile is not exactly clear.

A recent review by Spalart (1998), published 34 years after Batchelor's initial study, brings us a little closer to understanding how some test conditions affect the axial velocity phenomenon reported by Batchelor (1964) and subsequent experimental studies. In this report, Spalart (1998) sheds new light on Batchelor's analysis of a trailing line vortex and introduces his own analysis showing the interaction between the axial and 
tangential velocities. From his analysis, Spalart (1998) derives a proportional relationship between the axial velocity component, $\mathrm{U}_{\mathrm{x}}$, and a dimensionless quantity identified as the wing loading parameter, $\Gamma /\left(\mathrm{U}_{\infty} b\right)$, where $\Gamma$ is the wing circulation, $\mathrm{U}_{\infty}$ is the free stream velocity, and $b$ is the wing span. Using this wing loading parameter, Spalart (1998) proposes a way to reconcile the differences between experimental studies. Calculating this parameter for the test wings of Chow et al. (1997) and Green (1995), whose results show a "jet-like" maximum axial velocity of $1.78 \mathrm{U}_{\infty}$ and $1.62 \mathrm{U}_{\infty}$, gave $\Gamma /\left(\mathrm{U}_{\infty} \mathrm{b}\right)=0.20$ and 0.14 respectively. In contrast, Devenport et al. (1996), who reports a "wake-like" profile, has a much lower wing loading parameter $\Gamma /\left(U_{\infty} b\right)=0.028$. Since the wing loading parameter is strongly tied to the angle-of-attack and aspect ratio of the wing section, it is likely that the axial velocity profile is most significantly affected by these two parameters.

Five separate studies by Chigier and Corsiglia (1972), Corsiglia et. al (1973), Logan (1971), Orloff (1974), and McAlister and Takahashi (1991) confirm the strong relationship between the wing angle-of-attack and the axial velocity profile. Their results indicate a point of transition from "jet-like" to "wake-like" profiles for decreasing anglesof-attack. Even though the exact point of this transition is different for all five studies, the results follow the same trends predicted by the wing loading parameter. The study by McAlister and Takahashi (1991) also shows a strong relationship between the wing aspect ratio and the axial velocity profile. Under the experimental conditions where the Reynolds number, wing angle-of-attack, and chord length are held constant, data was collected for an aspect ratio of 6.6 and 8.1. The axial velocity profiles for these two wing configurations show a "jet-like" and "wake-like" profile, respectively. By calculating the wing loading parameter for each of these wing configurations and comparing the results to the respective axial velocity profiles, McAlister and Takahashi's (1991) data is again shown to be consistent with trends predicted by the wing loading parameter. 
These five studies, however, test no more than four angles-of-attack, three locations downstream of the wingtip, two different aspect ratios, and two different endcap treatments, none of which overlap with each other. This fact alone makes it impossible to conclusively determine which parameters have the most influence on the maximum axial velocity profiles. In fact, Green (1995) suggests that the axial velocity is strongly dependent on a different parameter, the Reynolds number, but admits that no one has yet given adequate evidence supporting this relationship. Nevertheless, a combination of these wing parameters and configurations, represented by the wing loading parameter, is a good predictor of the general behavior of the axial velocity in a wingtip vortex and can be used to reconcile the differences found in many experimental and numerical studies. 


\section{CHAPTER 2}

\section{EXPERIMENTAL FACILITY}

All experimental data were collected from the velocity measurements and flow visualization tests were performed in Utah State University's subsonic wind tunnel. This facility has a 4 -ft. $\times 4$-ft. square test section that extends 47 feet downstream from a 9:1 contraction ratio inlet to a 125 -foot long round diffuser. Several sides of the test section are made from 1-inch thick Plexiglas, which allow the observer to see the test model and equipment, and visually monitor the test conditions and procedures. Figure 2 shows a side and top view of the complete tunnel facility.

The wind tunnel test section can achieve a maximum speed of $123 \mathrm{mph}(55.0 \mathrm{~m} / \mathrm{s})$ with a maximum empty test section turbulence intensity level below $0.5 \%$. A 200 HP 3phase $\mathrm{AC}$ motor and a 6-foot diameter 4-blade variable pitch propeller pull the air through the test section. The speed of the motor is controlled with an Allen Bradley 1336VT variable frequency motor controller. The pitch of the propeller and the voltage input to the motor controller are remotely adjusted from within the control room. All equipment required to monitor the wind tunnel's operation and control the physical parameters of the study are located inside the control room.

Vital to accurate time averaged velocity measurements and precise flow seeding for visualization techniques is the quality of the airflow entering the inlet and being accelerated through the test section. A flow conditioning screen is located at the entrance of the inlet to reduce the overall turbulence level. This screen consists of 4.5 in. thick aluminum honeycomb. Each hexagonal cell of the honeycomb has a nominal size of $3 / 10^{-}$ inch diameter. An exploded schematic of the inlet assembly is shown in Figure 3.

The current study uses two Pentium II PC's and National Instruments' LabView and TSI's ThermalPro software to monitor atmospheric parameters and to control the 
tunnel speed. Each of these parameters is measured in real time and, if necessary, can be changed during the course of an experiment.

The parameters that are constantly monitored include the tunnel velocity, atmospheric pressure, and atmospheric temperature. The velocity is measured with a pitot static tube connected to a Setra Datum 2000 pressure transducer. The atmospheric pressure and temperature are monitored with a Setra model 276 barometric sensor and a type $\mathrm{T}$ thermocouple, respectively. The thermocouple circuit uses TSI's constant temperature anemometer as a cold junction reference. From these three parameters, LabView calculates the air density, sonic velocity, Mach number, and Reynolds number.

A NACA 0015 rectangular planform wing section with a 30 -inch chord and 24inch semi-span is used to generate the wingtip vortex. This model has a solid foam core cut to shape using a hot wire and two $1 / 8$-inch thick aluminum templates. These templates and a 2 -inch diameter aluminum pipe located at the quarter-chord of the wing make up the support structure of the model. Twelve layers of fiberglass composite and a painted finish protect the foam core and provide a smooth surface. Twenty-five pressure ports, twelve on each side and one at the leading edge, are positioned mid-span on the surface of the model. These ports are used to determine the angle-of-attack of the wing section by comparing the surface pressures on the suction and pressure sides of the wing during tunnel operation. A cut-a-way schematic of the wing structure is shown in Figure 4.

The finished wing section model is mounted vertically on a 40-in. $x 47$-in. splitter plate that extends horizontally between the wind tunnel walls. The upstream edge of the splitter plate is rounded to reduce the formation of turbulent eddies. Similarly, the downstream edge of the splitter plate is tapered to reduce the size of the wake it generates. The wing section is mounted such that its leading edge is 4 inches back from the nose and its trailing edge is 6 inches up from the tail of the splitter plate. Two aluminum tracks that have a 1 -in. $\times 1.5$ in. ' $U$ ' shaped cross-section are rigidly fastened 4 inches up from the wind tunnel floor. These tracks extend downstream 20 feet from the 
beginning of the test section and support the splitter plate and wing section. A picture of the splitter plate mount and the finished wing section is shown in Figure 5. The picture is taken looking downstream in the wind tunnel and shows the leading edge of the splitter plate and wing section. The aluminum tracks that support the splitter plate and wing section can also be seen in the bottom right and left-hand corners of the picture. The wing section shown is fitted with the flat end-cap. 


\section{CHAPTER 3}

\section{VORTEX STUDY ARRANGEMENT AND PROCEDURES}

The experimental setup for the current vortex study involves two separate surveys of the vortex structure. The first of these surveys uses a qualitative flow visualization technique to illuminate the major vortex structures and capture them on video footage and still images. The second survey uses a triple-sensor hot wire probe to survey the flow field in and around the developing vortex. The Reynolds number is held constant during the data collection process allowing a valid comparison of the results from the two surveys.

The flow visualization technique is quite sensitive to the flow conditions in the test section of the wind tunnel. Therefore, careful consideration to the atmospheric conditions and the velocity in the test section were taken. For example, if the tunnel speed is too slow, slight differences in the atmospheric conditions at either end of the tunnel cause noticeable unsteadiness in the test section and make precise placement of the smoke in the flow seeding process difficult. On the other hand, if the tunnel speed is too fast, the higher turbulence levels in the test section causes the smoke to diffuse more quickly leaving less to be entrained into the vortex structures and illuminated by the laser sheet. Based on these limitations, all flow visualization data was measured at an intermediate tunnel speed of $50 \mathrm{mph}(22 \mathrm{~m} / \mathrm{s})$, which gave the optimum flow visualization conditions and the highest quality video and still images. In addition, all velocity measurements were collected at this same speed so that an accurate comparison between the two surveys could be made. The Reynolds number of the flow in the test section based on the chord length of the wing section and for a velocity of $50 \mathrm{mph}$ is $1 \times 10^{6}$.

A global Cartesian coordinate system aligned with the fixed walls of the wind tunnel is also defined. This coordinate system aligns the positive $\mathrm{x}$-axis with the 
downstream direction of the wind tunnel parallel to the walls. The positive $y$-axis is directed upward perpendicular to the top and bottom of the wind tunnel walls and parallel to the span of the wing section. The right hand coordinate system rule then defines the positive $z$-axes to be directed to the left when facing upstream in the wind tunnel. The $\mathrm{x}$ and $\mathrm{y}$ directions in this coordinate system are often referred to as the streamwise and spanwise directions, respectively. The origin of this coordinate system is fixed to the wing section at the point of intersection between the trailing edge and the endcap. Locating the origin at this point means that it will move as the wing section is turned to different angles-of-attack. This coordinate system is used when collecting and reporting all flow visualization and velocity data.

Flow Visualization Setup

The flow visualization process for this study is separated into four different components, the flow seeding apparatus, the laser visualization apparatus, the recording apparatus, and the wing section model. The location of these components in relation to each other and the wind tunnel is shown in Figure 6.

Visualizing the development of the vortex structure began by seeding the flow upstream of the wing section model using titanium tetrachloride $\left(\mathrm{TiCl}_{4}\right)$ as described by Mueller (1996). The seeding process involved directly injecting neutrally buoyant smoke particles into the air stream with the use of a smoke rake. The smoke particles were generated from liquid titanium tetrachloride, which reacts with the water vapor in the air stream to form small titanium dioxide $\left(\mathrm{TiO}_{2}\right)$ particles and hydrochloric acid $(\mathrm{HCl})$ vapor. The $\mathrm{TiCl}_{4}$ is held in a glass Erlenmeyer flask that is connected with separate Tygon tubes to a pressurized tank of nitrogen $\left(\mathrm{N}_{2}\right)$ gas and to the smoke rake. Each tube is clamped near the inlet and outlet ports on the flask to control the infiltration of $\mathrm{N}_{2}$ gas into the flask and the expelling of the $\mathrm{TiCl}_{4}$ through the smoke rake. As desired, the clamps are released and the $\mathrm{N}_{2}$ gas forces the $\mathrm{TiCl}_{4}$ to sublimate and flow through the smoke rake 
into the airstream. The reaction process then occurs as the $\mathrm{TiCl}_{4}$ leaves the ports on the smoke rake forming a visible white smoke as it comes in contact with the air in the test section. Figure 7 shows a diagram of this smoke generation setup.

The purpose of the smoke rake is to direct individual streams of smoke particles into the shear layers that form on the pressure side of the wing. A hollow aluminum tube with a symmetric airfoil shaped cross section is the main structure of the smoke rake. Nine round exit ports located 1-inch apart are drilled along the trailing edge of the support tube. In order to avoid releasing the smoke directly into the wake of the support tube, each exit port is fitted with a $1 / 8$-inch diameter brass tube. These tubes extend 1 -inch downstream of the trailing edge of the support tube and deliver nine individual streams of smoke into the test section. A $300-\mathrm{mW}$ argon-ion laser is used to illuminate the smoke particles as they are entrained into the forming vortex structures.

A rail and slide assembly is mounted downstream of the argon laser to redirect the beam downward into the tunnel and expand the beam into a thin sheet. This assembly consists of a horizontal rail, a main vertical support post, which are used to position a 1.0-inch diameter broadband dielectric mirror and cylindrical lens. The rail and slide assembly is positioned on top of the tunnel according to Figure 8. The movement of the assembly along the horizontal slide controls the alignment between the laser beam, the mirror, and the cylindrical lens. The width of the laser sheet at the plane of intersection with the wingtip is approximately 10-12 inches allowing adequate coverage of the pressure and suction sides of the wing section. A highly polished portion of the Plexiglas wall between the lens and the wing section prevents distortion of the laser sheet as it passes into the tunnel.

Video images of the vortex structures were captured using a Pulnix TMC-7DSP CCD color camera with a Rainbow H6X8M-II $8-48 \mathrm{~mm}$ lens. The zoom, focus, and iris settings of the lens are motorized and adjusted from within the control room using a 
remote Rainbow A-III lens controller. A computer controlled pan-tilt unit adjusts the position of the camera and lens within the tunnel.

The pan-tilt unit and video camera are mounted to the inside top of the wind tunnel. The camera assembly is positioned in the upper right-hand corner of the tunnel if looking in the downstream direction. A sheet metal faring is also attached to the top of the tunnel and extends around the camera assembly. The purpose of the faring is to protect the camera assembly and to minimize the wake generated by the camera assembly. The position and relatively small size of the faring in relation to the wind tunnel crosssection helps minimize its effects on the uniform flow in the test section. In addition, the faring is sufficiently far away from the wing section model and generated vortex that it has no measurable effect on the flow visualization or velocity data.

\section{Velocity Measurements Setup}

The traversing apparatus provides the means of moving the probe in the yz-plane in the test section. The horizontal direction has a total range of 34 inches and the vertical direction has a range of 24 inches. The resolution of the horizontal and vertical stepper motors is 31250 and 125000 steps per inch, respectively. Communication with the motor controllers is achieved with TSI's ThermalPro software and is incorporated directly into the data acquisition system.

All velocity data collected for this study was obtained with an Auspex AVEP-3102 triple-sensor hot wire probe connected to a 5-channel TSI IFA300 constant temperature anemometer. The probe is capable of resolving the three components of velocity $\mathrm{u}, \mathrm{v}$, and $\mathrm{w}$ and has a small measurement area of approximately $0.0016 \mathrm{in}^{2}(1.0$ $\mathrm{mm}^{2}$ ). A diagram showing the specifications and dimensions of the triple-sensor probe is shown in Figure 9. Each sensor has a slant angle of 45 degrees and the azimuth angle between adjacent sensors is 60 degrees. Figure 10 shows the sensor array geometry of the Auspex triple-sensor probe. The triple-sensor probe is calibrated using the method of 
Lekakis et. al (1989) and the resulting velocity components are aligned with the wind tunnel coordinate system such that $\mathrm{u}, \mathrm{v}$, and $\mathrm{w}$, are the velocity components in the $\mathrm{x}, \mathrm{y}$, and $\mathrm{z}$ coordinate directions

The voltage time-series data from the anemometer is relayed to the computer through a 16-channel breakout board and a United Electronic Industries (UEI) data acquisition card. The data acquisition card is a 32-bit analog to digital converter with two 16-bit resolution inputs and outputs. 


\section{CHAPTER 4}

\section{DISCUSSION AND RESULTS}

The current study recorded flow visualization and measured velocity data at three streamwise locations $\mathrm{x} / \mathrm{c}=-0.2,0.05$, and 1.0 referenced from the trailing edge of the wing section. The data were collected at each location for; a constant Reynolds number of $1 \mathrm{x}$ $10^{6}$, angles-of-attack of $\alpha=4,8$, and 12 degrees, and. both the flat and round end-cap configurations. The mean freestream velocity for all data was $50 \mathrm{mph}(22 \mathrm{~m} / \mathrm{s})$. This velocity is used to normalize the axial and tangential velocities measured by the triplesensor probe and to calculate the non-dimensional loading parameter.

\section{Velocity Measurements Results}

The velocity profiles for the flat and round end-cap treatments are shown as contour plots where each contour line represents a constant axial or tangential velocity. The shaded regions shown in the contour plots represent invalid data locations where the flow angles exceeded its measurement capabilities triple-sensor probe. The physical geometry of the sensor array determines the measurement capabilities of the probe, and in the case of this study, limits the probe's ability to measure flow angles higher than 30 degrees. Outside of the shaded regions the data is valid and contains enough detail so that reasonable comparisons can be made between the velocity and flow visualization results and with the results from other wingtip vortex studies. The normalized increments between constant axial and tangential velocity contours are represented with the symbol $\Delta$, as seen just below each figure. The contour plots created for a streamwise location of $\mathrm{x} / \mathrm{c}=-0.20$ include an outline of the wing section to show its relative position with respect to the measurement plane. The region to the left of this outline is the suction side of the wing section and the region to the right is the pressure side of the wing section. The gap between the wing section outline and the velocity contours shown in the plots is 
the distance of the closest approach between the triple-sensor probe and the surface of the wing section, which was approximately _-inch for all angles-of-attack and end-cap treatments.

\section{Flat End-Cap Results}

\section{Degrees Angle-of-Attack}

The velocity contours for the wing section fitted with the flat end-cap at $\alpha=4$ degrees are shown in Figure 11a-f. The approximate center of any discernible vortex structure is identified with a letter such as A, B, C, etc. The relative movement of each vortex structure with respect to the wing section and other vortices cannot be determined from the presented velocity contours, but was determined from the video recordings taken during the flow visualization part of this study. Based on the information presented by the video recordings, the movements of each vortex structure is traced from one measurement plane to another and identified with the appropriate corresponding letter. In some instances, these same identifiers are used to label different parts of velocity profile plots that correspond to the location and characteristics of specific vortex structures.

The first two plots shown in Figure 11a,b indicate the presence of two distinct vortex structures that began their formation upstream of the velocity measurement plane located at $x / c=-0.20$. In both cases, the vortex structures appear to have formed at the sharp edges of the flat end-cap, which are natural separation points for the fluid as it accelerates around the end-cap from the pressure side to the suction side of the wing section. These two distinct vortex structures provide evidence that at this streamwise location a portion of the wing section's vorticity has been shed and is rolling-up into at least two separate vortices. The tangential and axial contours indicated obvious differences in the structure of each vortex. In general, these differences are characterized 
by higher tangential and axial velocity gradients found in one vortex over another. When these velocity gradients (axial and tangential) are localized around a common point they define an individual vortex structure, or what is termed as a "coherent" vortex. The relative magnitude of the velocity gradients around a particular vortex compared to another is what determines their comparative strength. Based on these definitions, the tangential and axial velocity contours in Figure $11 \mathrm{a}, \mathrm{b}$ indicate that the vortex on the suction side (positive $\mathrm{z} / \mathrm{c}$ ) of the wing section is stronger and more coherent than the vortex forming over the end-cap.

Figure $11 \mathrm{c}, \mathrm{d}$ shows the tangential and axial velocity contours for a streamwise location of $x / c=0.05$. The measurement plane for this location is slightly downstream of the trailing edge and thus provides a view of the vortex structures after leaving the region of direct interaction with the wing section. At this point in the flow field, all of the vorticity generated by the wing section has been shed into either the wake region, or rolled-up into the wingtip vortex structures. Even at this early stage of development, Francis and Kennedy (1979) reported that as much as half of the generated vorticity is contained in the wingtip vortex structures.

The tangential velocity contours in Figure 11c show that the two vortex structures have moved in a counter-clockwise ( $\mathrm{CCW}$ ) direction, as seen in the video footage, with respect to the wing section and are now aligned vertically in the y-direction with each other. Compared to their positions in Figure 11a,b, both vortex structures have moved inboard (negative y-direction) with respect to the wing section. At this point in the flow field, the velocity gradients of the two structures have somewhat been balanced making the features of the two vortices less unique compared to each other. Nonetheless, the relative locations of vortices $\mathrm{A}$ and $\mathrm{B}$ are consistent with those seen in the video footage.

The axial velocity contours presented in Figure 11d indicate the presence of four distinct regions of large axial flow gradients. These regions are identified with the lower case letters $a, b, c$, and $d$. The two upper regions (b and c) show relatively large axial 
velocity gradients and correspond to a location that is directly behind the tip of the endcap at the trailing edge of the wing section. The characteristics of the flow in this region are strongly influenced by both the pressure gradient from the end-cap and the merging wake at the trailing edge. The combination of those two factors produce a region of accelerated axial flow at the tip of the trailing edge. The next region corresponds to the center of rotation of vortex B identified in Figure 11c. The characteristics of this region help identify vortex $\mathrm{A}$ as being single coherent vortex. The final distinct region of axial flow shown in Figure $11 \mathrm{~d}$ is the trailing edge wake. This region is common to all velocity contours plots at $x / c=0.05$, which should be expected due to the close proximity to the trailing edge. As we will see in the contour plots for $\mathrm{x} / \mathrm{c}=1.0$, this region becomes less distinct further downstream as it is entrained by the dominant wingtip vortex.

The velocity contours for the last streamwise location of $x / c=1.0$ are shown in Figure 1 le,f. At this distance downstream of the trailing edge, both the tangential and axial velocity contours show that the two vortex structures, A and B, have merged into a single, relatively symmetric vortex. The rotational center of this vortex is located inboard (negative $\mathrm{y}_{-}$) and to the suction side (positive $\mathrm{z}-$ ) with respect to the tip of the trailing edge. The size of the surveyed region is not large enough to conclusively determine if the vortex roll-up is complete.

\section{Degrees Angle-of-Attack}

Figure 11 a-f shows velocity contours for the flat end-cap wing section positioned at an angle-of-attack of 8 degrees. Figure 12a,b show that for this increased angle-ofattack at least four, possibly five, vortex structures form upstream of the measurement plane located at the streamwise position of $x / c=-0.20$. Two of the vortex structures (D and $\mathrm{E}$ ) are formed along the pressure and suction side edges of the flat end-cap while the third, fourth, and possibly the fifth vortex structures (A, B, and C) are formed away from the surface on the suction side of the wing section. The features of each vortex structure 
become more distinct as one moves around the end-cap from the pressure side to the suction side of the wing section. In fact, it appears that vortex $E$ on the pressure edge of the end-cap is in the beginning stages of development. It is further apparent from the contour gradients shown in both the tangential and axial plots that vortex A, which formed away from the surface on the suction side of the wing section, is much stronger and more coherent than the other vortices. This is similar behavior to that described in the $\alpha=4$ degree results. The fact that the central region in the suction side vortex is shaded (indicating high flow angles) also shows that the strength of this vortex is greater in comparison to the other vortices. Furthermore, the larger flow angle encountered for the $\alpha=8$ degrees case compared to the $\alpha=4$ degrees case suggests that the strength of the suction side vortex is increasing with angle-of-attack. Also similar to the $\alpha=4$ degrees case, the formation of multiple vortex structures suggests that the wing section's vorticity is being shed from the wingtip in multiple sheets.

Advancement of the forming vortex structures to a streamwise location just behind the trailing edge of the wing section at $x / c=0.05$ is shown in Figure $12 \mathrm{c}, \mathrm{d}$. The velocity contours for this location show similar trends in the movement and coherence of the vortex structures as was described for the $\alpha=4$ degrees case. Identifying the new relative positions of each vortex labeled in Figure 12a,b indicate that they progress in a CCW direction with respect to the wing section from $\mathrm{x} / \mathrm{c}=-0.20$ to $\mathrm{x} / \mathrm{c}=0.05$. At this streamwise location, all of the vorticity generated by the wing section is contained in the wake region or in the individual vortices. Since the vortices are no longer being fed new vorticity, they begin to redistribute the existing vorticity between themselves causing their individual swirl velocities become nearly equal. Once the vortices move beyond the trailing edge, it is easier to identify the fourth vortex structure, B, located at the approximate center of rotation for the other four vortices. The contours for vortices A, $\mathrm{D}$, and $\mathrm{E}$ show that their individual tangential and axial velocity gradients correlate quite 
well. However, the tangential velocity gradients of vortices $\mathrm{B}$ and $\mathrm{C}$ do not correlate with a region of large axial velocity gradients.

Figure $12 \mathrm{e}, \mathrm{f}$ show velocity contours at a streamwise location of $\mathrm{x} / \mathrm{c}=1.0$. The tangential and axial velocity contours show that the vortex structures identified upstream have merged into one distinct vortex. However, this distinct vortex is more asymmetric than that shown in the $\alpha=4$ degrees case. This lack of symmetry indicates that the vortex structure for $\alpha=8$ degrees is not completely rolled-up at one chord length downstream. The contours further indicate that the single vortex structure has a much higher tangential and axial velocity gradients compared to the vortex at $\alpha=4$ degrees. Since the scale of the plots for both angles-of-attack are the same, the vortex generated at $\alpha=8$ degrees is more concentrated and has greater strength.

\section{Degrees Angle-of-Attack}

Tangential and axial velocity contours for the flat end-cap wing section positioned at an angle-of-attack of $\alpha=12$ degrees are shown in Figure 13a-f. Similar to the contour plots presented in Figures 11 and 12, these plots show the formation and progression of the wingtip vortex from a streamwise location of $x / c=-0.20$ to $x / c=1.0$. The contours in Figure 13a,b show the initial development of approximately five vortex structures. The tangential contours, Figure 13a, show that three of the five structures are reasonably distinct and form in similar locations with respect to the wing section as those measured at the lower angles-of-attack. The axial velocity contours shown in Figure 13b provide more definition to these three vortices and offer evidence of the other two, which form close to the suction side surface of the wing section. Again, it can be seen from both contour plots that the strongest vortex is located off of the surface on the suction side of the wing section.

The velocity contours at a streamwise location of $\mathrm{x} / \mathrm{c}=0.05$ are shown in Figure $13 \mathrm{c}, \mathrm{d}$. These plots show the relative positions of all five vortex structures identified in 
Figure $13 \mathrm{a}, \mathrm{c}$ after they have moved beyond the trailing edge. The same $\mathrm{CCW}$ processions of the vortices as seen at the lower angles of attack were observed with the aid of the video recording. Similar to the $\alpha=8$ degrees case, a distinct vortex structure is located at a central location with respect to the other vortices. This central vortex lacks the large tangential and axial velocity gradients that characterize the surrounding vortices.

The velocity contours for the last streamwise location of $x / c=1.0$ are shown in Figure 13e,f. These velocity contours show that the individual vortices identified upstream have begun to merge into a single vortex. Similar to the plots shown for $\alpha=8$ degrees, this single vortex structure has a large region of high velocity gradients and flow angles. However, comparing the velocity gradients at $\alpha=12$ degrees with those seen at lower angles-of-attack suggests that the wingtip vortex is progressively getting stronger as the angle-of-attack is increased. Unlike the vortices shown at the lower angles-of-attack, this single vortex is more asymmetric and has evidence of several other distinct vortex structures indicating that the roll-up is finalized further downstream.

\section{Flat End-Cap Discussion}

The tangential and axial velocity contours for the flat end-cap wing section show multiple vortex structures forming at a streamwise locations of $\mathrm{x} / \mathrm{c}=-0.20$ for all three angles-of-attack. It was deduced from the video recording that the vortices observed at this streamwise location originate upstream along the edges of the flat end-cap. These edges, which act as natural points of separation, divide the vortex sheet being accelerated around the wingtip into multiple sheets that eventually form the vortex structures described by the velocity contours. The strength and coherence of the individual vortices is directly related to the location along the edge of the end-cap from which they separate. This is evident in the consistently stronger and more coherent suction side vortex that forms early (near the leading edge) on the suction side of the wing section as seen in the video footage and Figures 11-13. To illustrate this phenomenon, Figure 14a-c shows the 
front, top, and side views of the wing section fitted with the flat end-cap for all three angles-of-attack.

Each of the illustrations shown in Figure 14a-c represents the initial formation and progression of the vortex structures identified in the velocity contours of Figures 11-13. The general path each vortex follows in the illustrations is obtained from the video footage. Figure 14a shows that two vortices form near the leading edge of the wing section. Initially, due to a pressure difference across the two sides of the wing section, a single vortex begins to form along the pressure side edge of the end-cap. At first, the vortex is quite small and entrains only a portion of the fluid that is being accelerated around the wingtip. The remainder of the fluid moves over the top of the small pressure side vortex and separates along the suction side edge of the end-cap as shown in the front view of Figure 14a. As the vortices move downstream, the pressure difference across the end-cap increases causing the momentum of the fluid moving over the wingtip to increase and the vortices to progressively gain strength. Eventually, the vortex forming along the pressure side of the wing section becomes sufficiently large that it spans the local width of the end-cap and effectively hides the suction side edge. By doing so, it forces the vorticity being generated in the pressure side boundary layer to wrap around the end-cap to the suction side of the wing section and bypass the "hidden" edge of the end-cap. In general, this limits the amount of vorticity being fed to the vortex along the end-cap and increases the flow of vorticity to the suction side vortex. The result is a stronger more coherent suction side vortex as seen in Figures 11-13. The top/side view of Figure 14a shows this phenomenon with lines representing the path of the fluid being entrained into the vortices.

Figure 14b,c illustrates the same phenomenon shown for $\alpha=4$ degrees, but does so for $\alpha=8$, and 12 degrees. The primary difference between each illustration is the magnitude of the pressure gradient across the two sides of the of the wing section. This higher pressure gradient causes the initial pressure side vortex to migrate more quickly 
toward the suction side of the wing section as shown in the top/side view of Figure 14b,c. As this occurs, the pressure side vortex spans the width of the end-cap sooner causing the suction side vortex to entrain more vorticity at an early streamwise location and grow in strength more rapidly than any other vortex. In addition, as the suction side vortex moves toward the suction side of the wing section space becomes available along the pressure side edge of the end-cap where a new vortex can form.

Once the vortices leave the trailing edge of the wing section, the pressure gradients in the flow field begin to equalize and the velocity gradients become more uniformly distributed. The number and relative position of these individual vortices is clearly identified in Figures $11-13$ at $\mathrm{x} / \mathrm{c}=0.05$. Based on the mechanisms illustrated in Figure $14 a-c$, the number of vortices generated by the flat end-cap increase with an increase in the angle-of-attack; thus two vortices are generated at $\alpha=4$ degrees, four at $\alpha=8$ degrees, and five at $\alpha=12$ degrees. Video footage confirms that these vortices move in a $\mathrm{CCW}$ direction with respect to the wing section, which is consistent with the direction of flow over the wingtip induced by the pressure differential. In addition, the video footage alludes to a claim that the multiple vortices generated at each of the angles-of-attack are rotating about a common center. The location of this common center is more discernible at the two higher angles-of-attack and is marked by its central location and velocity characteristics compared to the other vortex structures. Vortices B in Figures $12 \mathrm{c}$ and $13 \mathrm{c}$ are considered to be the center of rotation for the surrounding vortex structures. Partial evidence of this claim is shown in the tangential and axial velocity contours for vortices B. These contours show relatively low tangential and axial velocity gradients in the region surrounding the two vortices. These velocity contours are not consistent with the distinct individual vortices present in the surrounding regions, which suggests that the central vortices were not formed by the same mechanisms that created the stronger surrounding vortices, but instead were formed as a result of the rolling-up process. 
More conclusive evidence to the claim that the vortices rotate in a $\mathrm{CCW}$ direction about a common center is shown in Figure 15a-c. This figure shows the spanwise axial velocity profiles for three different $y / c$ locations cut horizontally through Figure $13 \mathrm{~d}$. Figure $15 \mathrm{a}, \mathrm{b}$ indicates the existence of three relatively strong vortex structures $\mathrm{A}, \mathrm{C}$, and $\mathrm{D}$, where the spanwise velocity in the center of each vortex is approximately zero. Figure $15 \mathrm{c}$ indicates that a fourth vortex structure, $\mathrm{E}$, exists, but that it is relatively weak compared to the other three vortices. Considering the direction of the spanwise velocity for all data points surrounding vortices $\mathrm{A}, \mathrm{D}$, and $\mathrm{E}$, we see that their general motion is up or in the positive $\mathrm{y} / \mathrm{c}$ direction according to Figures $13 \mathrm{~d}$ and $15 \mathrm{a}-\mathrm{c}$. Furthermore, the general motion of vortex $\mathrm{C}$ is in the downward or negative $\mathrm{y} / \mathrm{c}$ direction according to the same Figures. Between these opposite moving vortices, Figure 15a shows a line of data points that pass through zero, where the points on the right are moving upward and the points on the left are moving downward. The zero point on this line corresponds to the vortex $\mathrm{B}$ in Figure 13d and is the center of rotation for vortices $\mathrm{A}, \mathrm{C}, \mathrm{D}$, and $\mathrm{E}$.

\section{Round End-Cap Results and Discussion}

The tangential and axial velocity contours for the round end-cap wing section at $\alpha$ $=4,8$, and 12 degrees are shown in Figures 16-18. The data for these contours were collected at the streamwise locations of $x / c=0.05$ and $x / c=1.0$. Due to high flow angles over most of the measurement plane, the contour plots of the data collected at $x / c=-0.15$ are not presented. Flow visualization and the video footage for this streamwise location, however, show that the flow moves smoothly around the end-cap and does not begin to separate into a distinct vortex until approximately $\mathrm{x} / \mathrm{c}=-0.30$. This is in contrast with the flat end-cap results, which show the initial formation of the first vortex to be near the leading edge. Nevertheless, the velocity contours shown for the other streamwise locations hold sufficient information to describe the structure of the wingtip vortex generated with a round end-cap. 
Figure 16a-d shows the velocity contours at $\alpha=4$ degrees. Unlike the multiple vortices shown in the flat end-cap results, the round end-cap velocity contours show a single coherent vortex with relatively high tangential and axial velocity gradients. Figure $16 a, b$ shows that the structure of the vortex just behind the trailing edge is nearly symmetric with moderate influence from wake region that is beginning to be entrained into the vortex structure. Noting that the scale of the axes in Figures $11 \mathrm{c}, \mathrm{d}$ and $16 \mathrm{a}, \mathrm{b}$ is the same, a comparison between the two figures shows that the single vortex generated from the round end-cap is larger than the two vortices generated by the flat-end-cap. This is expected since the vortex generated from the round end-cap contains nearly the same amount of the wing section's vorticity as does the two vortices generated from the flat end-cap. Further downstream at $\mathrm{x} / \mathrm{c}=1.0$, the contours of Figure $16 \mathrm{c}, \mathrm{d}$ indicate that the roll-up of the vortex is nearly complete and very little influence from the wake region remains. Moreover, the velocity contours remain relatively concentrated, especially in the axial direction, compared to the velocity contours shown in Figure 11e,f.

The velocity contours for $\alpha=8$ degrees are shown in Figure 17a-d. These contours show two vortex structures forming next to each other that persist from the measurement plane located at $\mathrm{x} / \mathrm{c}=0.05$ to $\mathrm{x} / \mathrm{c}=1.0$. Comparing the larger structure with the vortex seen at $\alpha=4$ degrees, we see that an increase in the angle-of-attack has increased the size and strength of the vortex. Flow visualization footage indicates that the larger vortex originated along the round end-cap, but does not show where the second much weaker vortex originated. The velocity contours at $x / c=0.05$, shown in Figure $17 \mathrm{a}, \mathrm{b}$, seem to indicate that it is formed due to some interaction between the wake region and the stronger vortex. Though the influence of the wake region at $\alpha=4$ degrees is relatively small by one chord length downstream, Figure $17 \mathrm{c}, \mathrm{d}$ shows that it continues to have a noticeable influence on the structure of the vortex in the form of the much smaller vortex present at $\mathrm{x} / \mathrm{c}=0.05$. 
The velocity contours for $\alpha=12$ degrees are shown in Figure 18a-d. Similar to the $\alpha=8$ degrees case, two vortices have formed just downstream of the trailing edge. Both vortices have increased in size and strength from those seen at $\alpha=8$ degrees and continue to remain nearly unchanged to $x / c=1.0$. The video footage indicates that the larger vortex structure originated upstream of the trailing edge on the suction side of the end-cap, but does not conclusively show where the smaller vortex originated or even that it persists downstream to $\mathrm{x} / \mathrm{c}=1.0$.

\section{Loading Parameter Analysis}

One of the goals of this study was to reconcile the different axial velocity profiles presented by previous experimental and numerical studies. As suggested by Spalart (1998), a significant interplay between the tangential and axial velocity components can lead to cases where both a "wake-like" and "jet-like" axial velocity profile can occur in vortices generated by the same wing section and end-cap treatment. In his analysis, Spalart (1998) derives a proportional relationship between the axial velocity component, $U_{x}$, and a dimensionless quantity identified here as the wing loading parameter, $\Gamma /\left(U_{\infty} b\right)$, where $\Gamma$ is the wing circulation, $U_{\infty}$ is the free stream velocity, and $b$ is the wing span. It is theorized that this loading parameter can be used to determine the magnitude of the Bernoulli effect on the characteristics of the axial flow.

Using the velocity data from the current study, the loading parameter for each wing configuration and all three angles-of-attack was calculated by integrating the tangential velocity components around the edges of the measurement plane located at $x / c$ $=1.0$. The loading parameter results are shown in Figure 19, which is a plot of the normalized axial velocity component near the center of the vortex versus the corresponding loading parameter. Also shown in this figure are results from studies performed by Chow et al. (1997), Dacles-Mariani et al. (1995), Devenport et al. (1996), and Green (1995). The axial velocity profiles used to generate Figure 19 are presented in 
Figure 20. The loading parameter results indicate that a "jet-like" profile is obtained when the loading parameter is on the order of 0.1 or greater, and a "wake-like" profile is obtained when the loading parameter is on the order of 0.1 or less. The dotted horizontal and vertical lines in Figure 19 represent the region of transition from a "wake-like" to a "jet-like" profile at $\Gamma /\left(\mathrm{U}_{\infty} b\right)=0.1$. Since all of the reported values fall within the first or third quadrants of the graph, it is concluded that the loading parameter is a good indicator of when a "jet-like" or a "wake-like" axial velocity profile can be expected.

The loading parameter illuminates an additional effect that the end-cap treatments have on the structure of the generated vortex. Figure 19 shows that the loading parameter calculated for the wing section fitted with the flat end-cap is consistently higher for each angle-of-attack, compared to the round end-cap. However, Figure 19 shows that the round end-cap wing section produces a stronger "jet-like" and "wake-like" behavior compared to the flat end-cap. This seemingly contradictory result is explained by the pressure data from McAlister and Takahashi (1991) and from the velocity data collected in this study. The data from McAlister and Takahashi (1991) shows that the flat end-cap produces a slight increase the lift and thus the loading on the wingtip of the wing section. The velocity data from this study shows that the vorticity is more concentrated in the vortex generated from the round end-cap causing an increase in the Bernoulli effect, or the interaction between the tangential and axial velocity components.

Flow Visualization Results

The results from the flow visualization study are shown in Figures 21-26. These figures include the flat and round end-cap treatments, all three angles-of-attack, and the measurement planes, $\mathrm{x} / \mathrm{c}=-0.20$ (flat end-cap), $\mathrm{x} / \mathrm{c}=-0.15$ (round end-cap), $\mathrm{x} / \mathrm{c}=0.05$, and $\mathrm{x} / \mathrm{c}=1.0$. The flow parameters, angle-of-attack of the wing section, and streamwise test locations for the visual survey were consistent with those used in the velocity survey to allow a direct comparison and facilitate an accurate interpretation of the two sets of 
results. It should be noted that the orientation of the images shown in Figures 21-26 are from a vantage point upstream of the measurement plane as opposed to the downstream view seen in the velocity contours. Because of this reversed vantage point, the pressure side of the wing section is located on the left in the images and the suction side is located on the right. The images were captured from the video footage recorded during the flow visualization survey of the wingtip vortex. The still images of the wingtip vortex provide a good representation of the vortex structure, however, they provide only limited information regarding the vortex dynamics. By comparison, watching the video footage allows the viewer's eyes to integrate the series of captured images over time introducing more detail and better resolution of the vortex dynamics. Therefore, reference to the information contained in the video footage will be included in the discussion of the images shown in Figures 21-26 to provide a more complete look at the formation and development of the wingtip vortex.

\section{Flat End-Cap}

Figure $21 \mathrm{a}-\mathrm{c}$ shows the flow visualization images for the flat end-cap wing section at $\alpha=4$ degrees. At this angle-of-attack, Figure 21a shows that two distinct vortex structures have formed, one located over the end-cap and the other just below the end-cap on the suction side of the wing section. These two vortices are stable in time showing only slight unsteadiness attributed to the flow conditions in the test section and not to the nature of their formation. The vortex located over the end-cap began its formation near the leading edge of the wing section and continued along the pressure side edge of the flat end-cap to the location shown. Similarly, the suction side vortex began to form near the leading edge, but left the edge of the end-cap shortly after its initial formation and moved downward in the images toward the root of the wing section. The progression of these two vortices as they move downstream is illustrated in Figure 14a-c. 
Figure $21 \mathrm{~b}$ shows that as the vortices move further downstream to $\mathrm{x} / \mathrm{c}=0.05$ they process in a clock-wise $(\mathrm{CW})$ direction with respect to the wing section. Due to the reversed vantage point, this is consistent with their direction of rotation as seen in the velocity contours. The image indicates that the vortices are vertically aligned with each other and the trailing edge of the wing section with a hint of the wake region being entrained in to the top structure. This is consistent with the velocity contours shown in Figure $11 \mathrm{c}, \mathrm{d}$. As the vortices continue moving downstream in the test section, the video footage shows that they process around each other in a helical pattern until they eventually merge into a single vortex. Figure $21 \mathrm{c}$ shows the final stages of this merging process at the streamwise location of $x / c=1.0$. Though, the flow visualization images indicate that the roll-up process is complete by $\mathrm{x} / \mathrm{c}=1.0$, the velocity contours contradict this conclusion by showing the existence of small structure that are not completely entrained in to one vortex. This discrepancy is due to the smoke becoming more diffuse as it moves further downstream resulting in a lower resolution of the images.

Figures 22a-c shows the development of the wingtip vortex from $\mathrm{x} / \mathrm{c}=-0.02$ to $\mathrm{x} / \mathrm{c}$ $=1.0$ for $\alpha=8$ degrees. Confirming the result shown in the contour plots of Figure 12, the flow visualization images show three vortex structures forming adjacent to the flat end-cap and processing in a $\mathrm{CW}$ direction downstream until they merge into a single vortex. Similarly, Figure $23 a-c$ shows three vortex structures being generated by the flat end-cap that move downstream in a helical pattern until the merge. A comparison of the velocity contours in Figures 12a,c and 13a,c with the flow visualization images in Figures $22 a, b$ and $23 a, b$ show that the locations of the structures in each measurement correlate well. This correlation provides confidence in the results of both surveys.

\section{Round End-Cap}

The flow visualization images for the round end-cap wing section at all three angles of attack are shown in Figures 24-26. All images show that a single vortex is 
generated on the suction side of the wing section no further upstream than $\mathrm{x} / \mathrm{c}=-0.60$. Unlike the fixed separation point caused by the edges of the flat end-cap, the single vortex generated by the round end-cap is formed strictly by the pressure gradient between the surfaces of the wing section. The video footage shows that without a fixed separation point the initial formation of the wingtip vortex is delayed. Though the exact point of formation cannot be determined by the video, it is clear that formation occurs sooner at higher angles-of-attack or stronger pressure gradients. Once the single vortex forms, it remains in a region near the end-cap on the suction side of the wing section, which is dominated by a large flow of vorticity from the pressure to the suction side of the wing section. This result is similar to the phenomenon described for the strong suction side vortex generated by the flat end-cap geometry.

As the vortex moves beyond the trailing edge, a "mushroom shaped" section forms near the center of the vortex. This section is formed as the wake region interacts with the wingtip vortex during the initial stages of the roll-up process. This interaction becomes more noticeable at higher angles-of-attack as the as the fluid in the wake region is accelerated more quickly into the existing vortex structure. The influence of the wake, according to the flow visualization images, is progressively diminished for all three anglesof-attack until a single symmetric vortex exists at $\mathrm{x} / \mathrm{c}=1.0$. 


\section{CHAPTER 5}

\section{CONCLUSIONS AND FUTURE RESEARCH}

\section{Research Conclusions}

The structure and development of the wingtip vortex from a NACA 0015 wing section was investigated by surveying the flow field with triple-sensor hot wire velocity measurements and flow visualization images. The results from these surveys show that multiple vortex structures form next to the flat end-cap wingtip for each tested angle-ofattack of $\alpha=4,8$, and 12 degrees. Flow visualization images show that these vortices begin to form near the leading edge of the wingtip at the fixed separation point formed by the edges of the flat end-cap. The velocity and flow visualization data suggests that the edges of the flat end-cap divide the vorticity created by the wing section into multiple vorticity sheets. The divided vorticity sheets form individual, relatively strong vortices, which move downstream in a counter-clockwise direction around a common center until they eventually merge to form a single coherent wingtip vortex. The downstream distance required for the vortex structures to completely merge progressively increases for increasing angles-of-attack. The data also show that the number of individual vortices shed from the flat end-cap increases as the angle-of-attack increases from approximately two at $\alpha=4$ degrees to five at $\alpha=12$ degrees.

In contrast with the flat end-cap results, the velocity and flow visualization data show that a single coherent vortex forms on the suction side of the round end-cap for all three angles-of-attack. This single vortex remains relatively strong and coherent through the final measurement plane of $x / c=1.0$. The velocity measurements show that the tangential velocity component is higher in the single vortex produced by the round endcap as compared to multiple vortices produced by the flat end-cap. The difference in this component of velocity is attributed to the distribution of vorticity in the vortex structures generated from each of the end-cap treatments. The single vortex that is generated by the 
round end-cap concentrates the vorticity of the wing section into a tightly wound structure that produces a high tangential velocity. In contrast, the multiple vortices generated by the flat end-cap distribute the vorticity further from the common center of rotation, which produces a lower tangential velocity. In addition, the energy lost to the surrounding fluid during the merging process of the multiple vortices reduces the overall strength of the resulting single vortex and produces a relatively lower tangential velocity.

The ability of the loading parameter to reconcile differences in the axial velocity profiles from this study and previous experimental and numerical studies was evaluated. The results show that the loading parameter is a good indicator of when a "jet-like" or a "wake-like" axial velocity profile can be expected. The general trends from this study and previous studies show that a "jet-like" axial velocity profile can be expected for loading parameter values greater than 0.1 and that a "wake-like" profile can be expected for loading parameter values less than 0.1 . This parameter provides additional information that can help future experimental studies and numerical codes compare and validate their axial velocity results with existing axial velocity trends.

The magnitude of the loading parameter, however, cannot be used to predict the magnitude of the axial velocity excess or deficit based on the varying effects the geometry of the wing section and its end-cap have on the value of the loading parameter. For example, the flat end-cap produces lower jet velocities at each angle-of-attack while producing higher loading parameter values. The cause of this result is traced back to the initial formation of the vortices produced by each end-cap treatment. The multiple vortices generated by the flat end-cap form closer to the leading edge of the wing section, which causes a decrease in the pressure, an increase in the lift, and therefore, an increase in the loading parameter when compared to the round end-cap. However, the single vortex generated by the round end-cap is more concentrated and contains more energy, which increases the Bernoulli effect and is responsible for the higher jet velocity. 
The results from the flow visualization images confirm the presence of multiple vortices being generated from the flat end-cap and a single vortex being generated from the round end-cap. They also confirm that the vortex structures process downstream in a helical pattern until they merge into a single, nearly symmetric vortex. Low resolution in the images, however, prevents the fine details of the flow from being seen. Therefore, results from the velocity and flow visualization surveys are correlated in order to more clearly understand the structure and development of the wingtip vortex.

Future Research

The results of this study show general trends in the formation of a wingtip vortex generated from a flat and round end-cap. The details of this formation, such as the origin, the relative movements, and the structure of the core of each vortex were not presented. These fine details require data to be collected at more streamwise locations, particularly adjacent to the end-cap, and at more angles-of-attack. In addition, steps need to be taken to reduce the effects of high flow angles on the data. This may require a different probe that is less sensitive to high flow angles, a more precise calibration process for the hot wire probe, or a new technique in obtaining the velocity data such as a laser doppler velocimeter (LDV) or a multi-hole pressure probe. In addition to improving the velocity measurements, better flow visualization techniques should be investigated that will improve the flow seeding process, reduce the diffusion of the smoke after being entrained into the vortex, and improve the resolution of the images so that finer detail can be observed. 


\section{REFERENCES}

Arndt, R.E.A., V.H. Arakeri, and H. Higuchi. 1991. Some observations of tip-vortex cavitation. Journal of Fluid Mechanics 229: 269-289.

Batchelor, G.K. 1964. Axial flow in trailing line vortices. Journal of Fluid Mechanics 20: 645-658.

Chigier, N.A., and V.R. Corsiglia. 1972. Wind-tunnel studies of wing wake turbulence. Journal of Aircraft 9 (December): 820-825

Chow, J.S., G.G. Zilliac, and P. Bradshaw. 1997. Mean and turbulence measurements in the near field of a wingtip vortex. AIAA Journal 35 (October): 1561-1567.

Corsiglia, V.R., R.G. Schwind, and N.A. Chigier. 1973. Rapid scanning, threedimensional hot-wire anemometer surveys of wing-tip vortices. Journal of Aircraft 10 (December): 752-757.

Dacles-Mariani, J., G.G. Zilliac, J.S. Chow, and P. Bradshaw. 1995. Numerical/ experimental study of a wingtip vortex in the near field. AIAA Journal 33 (September): 1561-1568.

Devenport, W.J., M.C. Rife, Liapis, and Follin. 1996. The structure and development of a wing-tip vortex. Journal of Fluid Mechanics 312: 67-106.

Francis, M.S., and D.A. Kennedy. 1979. Formation of a trailing vortex. Journal of Aircraft 16 (March): 148-154.

Francis, T.B., and J. Katz. 1988. Observations on the development of a tip vortex on a rectangular hydrofoil. Journal of Fluids Engineering 110: 208-215.

Green, S.I., ed. 1995. Wing tip vortices. Fluid Vortices, 427-470. New York: Kluwer Academic Publishers.

Hsiao, C.T., and L.L. Pauley. 1996. Numerical study of the tip vortex flow over a finitespan hydrofoil. In ASME 1996 Fluids Engineering Division Conference 238: 65-74. 
Katz, J., and J.B. Galdo. 1989. Effect of roughness on rollup of tip vortices on a rectangular hydrofoil. Journal of Aircraft 26 (March): 247-253.

Lekakis, I.C., R.J. Adrian, and B.G. Jones. 1989. Measurements of velocity vectors with orthogonal and non-orthogonal triple-sensor probes. Experiments in Fluids 7: 228-240.

Liang, X., and B.R. Ramaprian. 1991. Visualization of the wing-tip vortex in temporal and spatial pressure gradients. Journal of Fluids Engineering 113: 511-515.

Logan, A.H. 1971. Vortex velocity distributions at large downstream distances. Journal of Aircraft 8 (November): 930-932.

McAlister, K.W., and R.K. Takahashi. 1991. NACA 0015 wing pressure and trailing vortex measurements. NASA Technical Paper 3151:1-136.

Mueller, T.J. 1996. Flow visualization by direct injection. Fluid Mechanics Measurements. 2d ed. ed. R.J. Goldstein, 367-450. Philadelphia: Taylor \& Francis.

Orloff, K.L. 1974. Trailing vortex wind-tunnel diagnostics with a laser velocimeter. Journal of Aircraft 11 (August): 477-482.

Ramaprian, B.R., and Y. Zheng. 1997. Measurements in rollup region of the tip vortex from a rectangular wing. AIAA Journal 35 (December): 1837-1843.

Rossow, V.J., J.N. Sacco, P.A. Askins, L.S. Bisbee, and S.M. Smith. 1995. Wind-tunnel measurements of hazard posed by lift-generated wakes. Journal of Aircraft 32 (March): 278-284.

Sarpkaya, T. 1998. Decay of wake vortices of large aircraft. In Proceedings of the AIAA $36^{\text {th }}$ Aerospace Sciences Meeting \& Exhibit held in Reno, Nevada 12-15 January 1998.

Shekarriz, A., T.C. Fu, J. Katz, H.L. Liu, and T.T. Huang. 1992. Study of junction and tip vortices using particle displacement velocimetry. AIAA Journal 30 (January): 145152.

Shekarriz, A., T.C. Fu, J. Katz, and T.T. Huang. 1993. Near-field behavior of a tip vortex. AIAA Journal 31 (January): 112-118. 
Spalart, P.R. 1998. Airplane trailing vortices. Annual Review of Fluid Mechanics 30: 107-138.

Thompson, D.H. 1975. Experimental study of axial flow in wing tip vortices. Journal of Aircraft 12 (November): 910-911. 


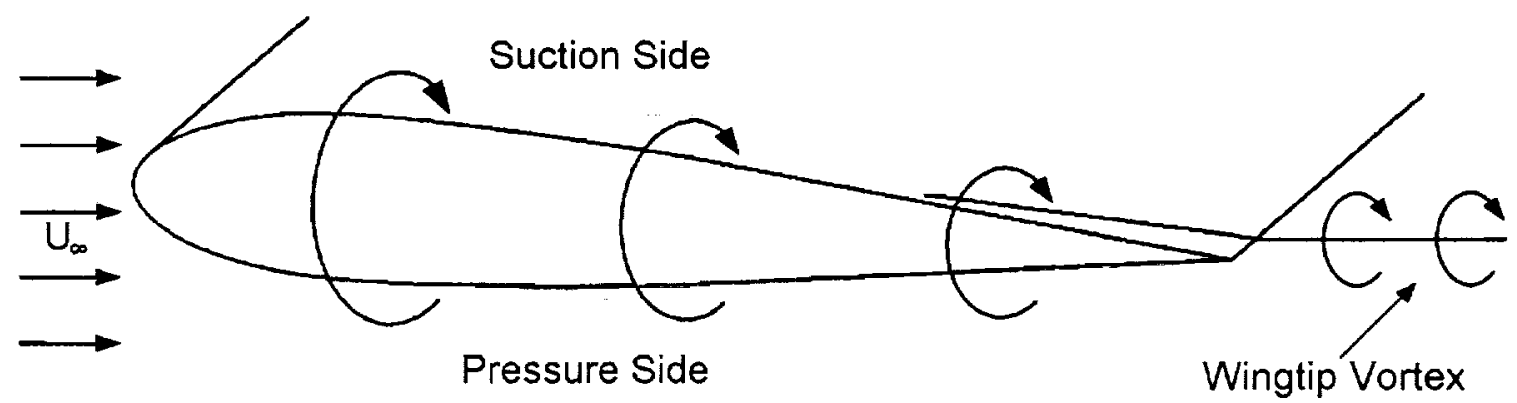

Figure 1. Wingtip vortex interpretation due to a pressure field around the wing.
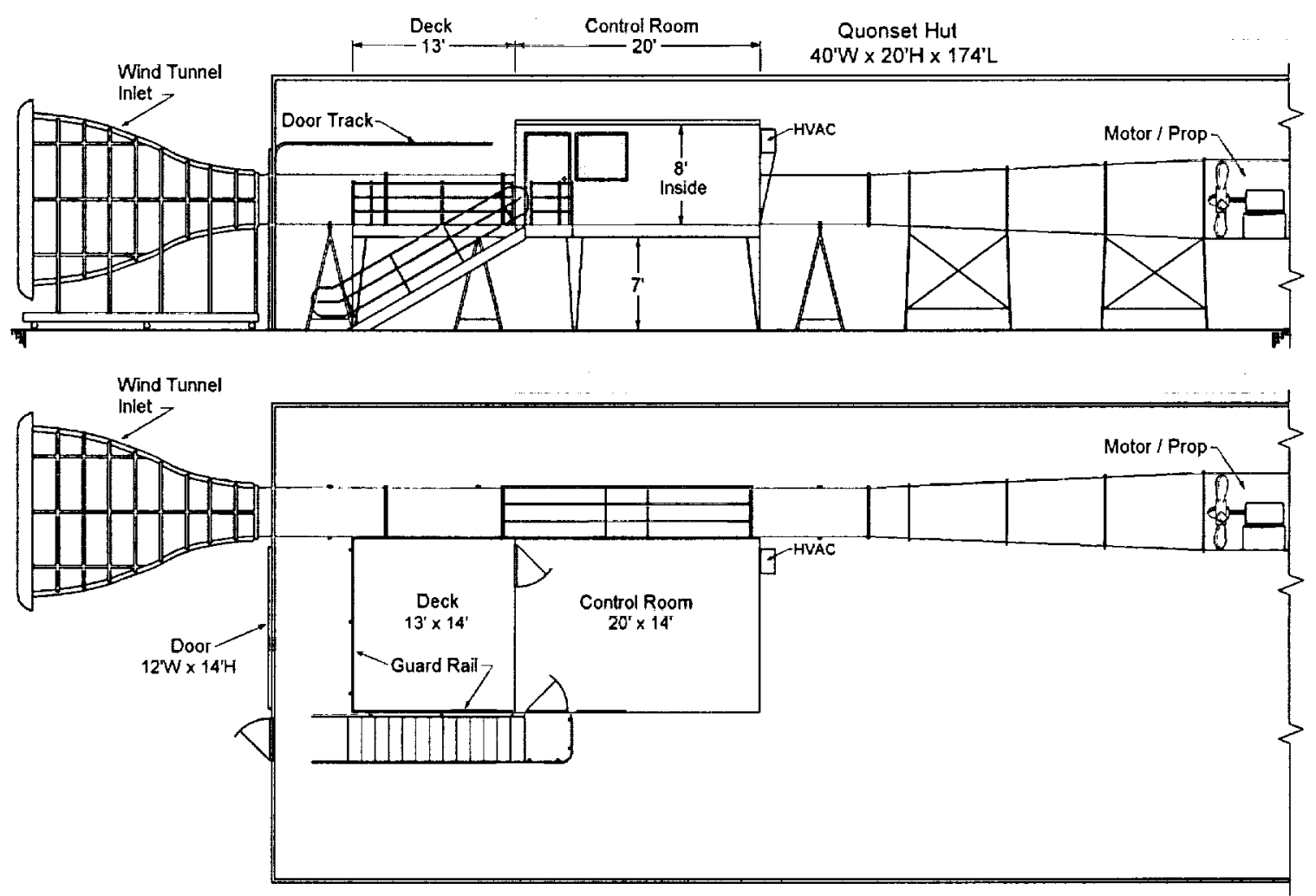

Figure 2. Side and top views of the complete wind tunnel facility. 


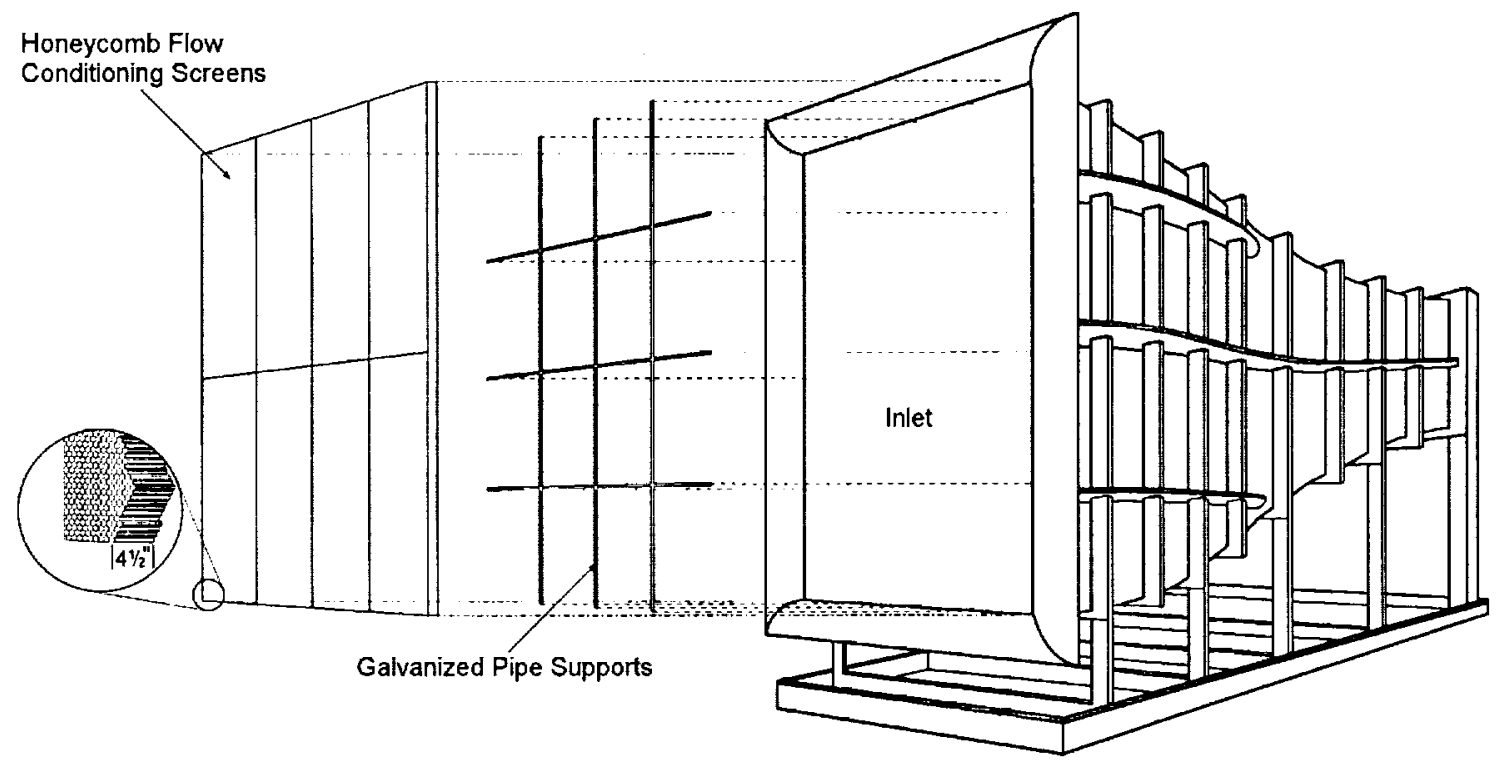

Figure 3. Exploded view of the inlet assembly.

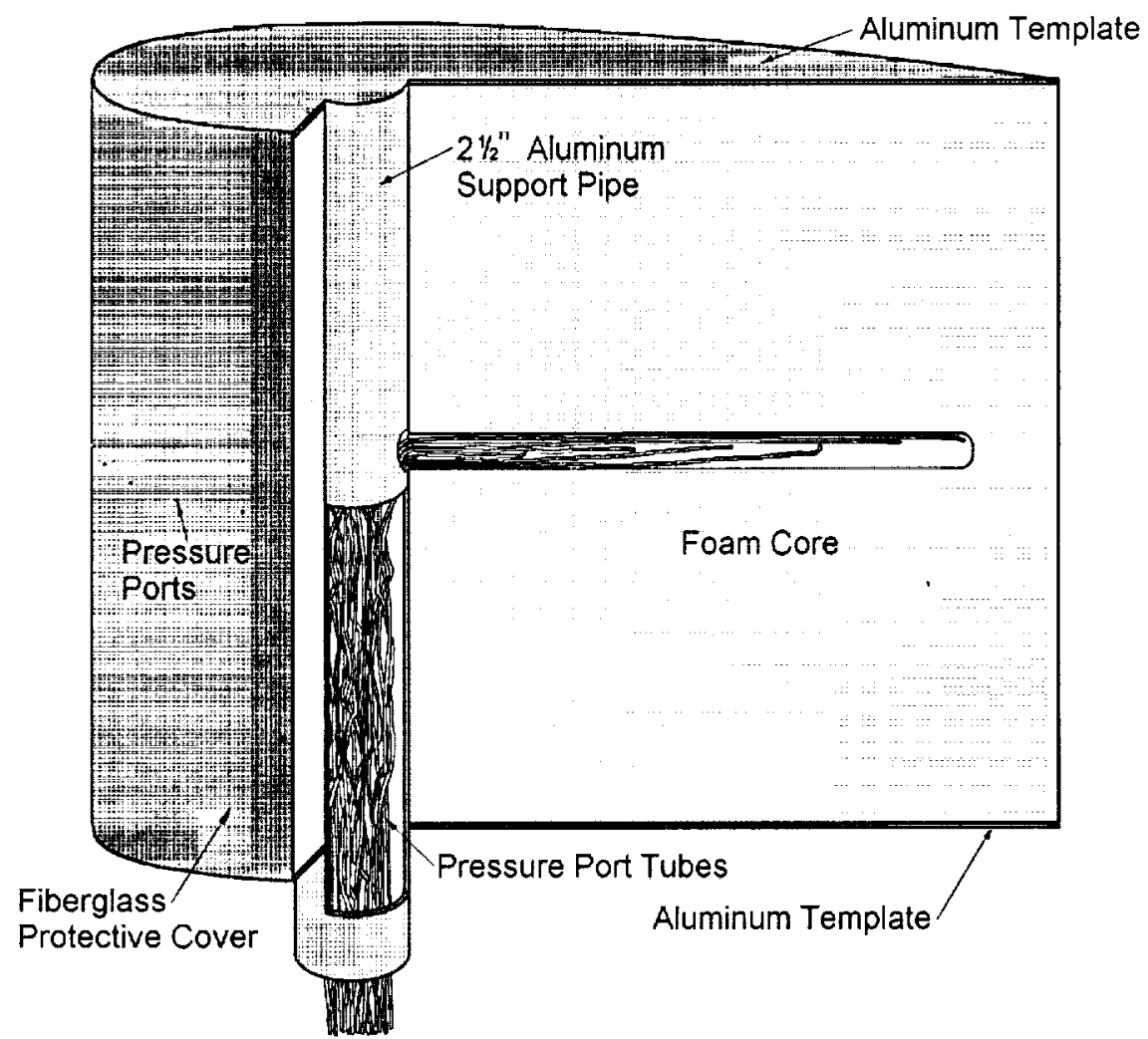

Figure 4. Cut-a-way schematic of the wing section structure. 


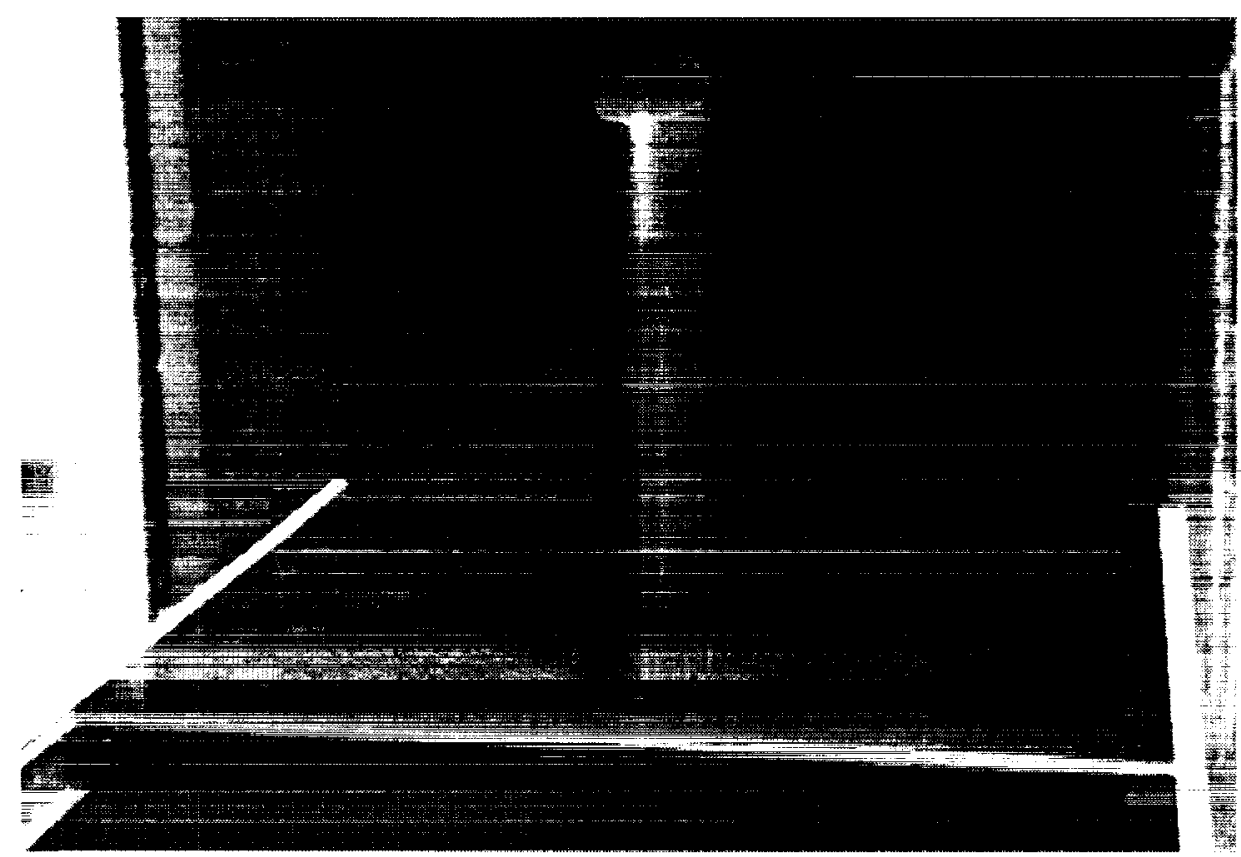

Figure 5. Wing section and splitter plate positioned in the wind tunnel test section.
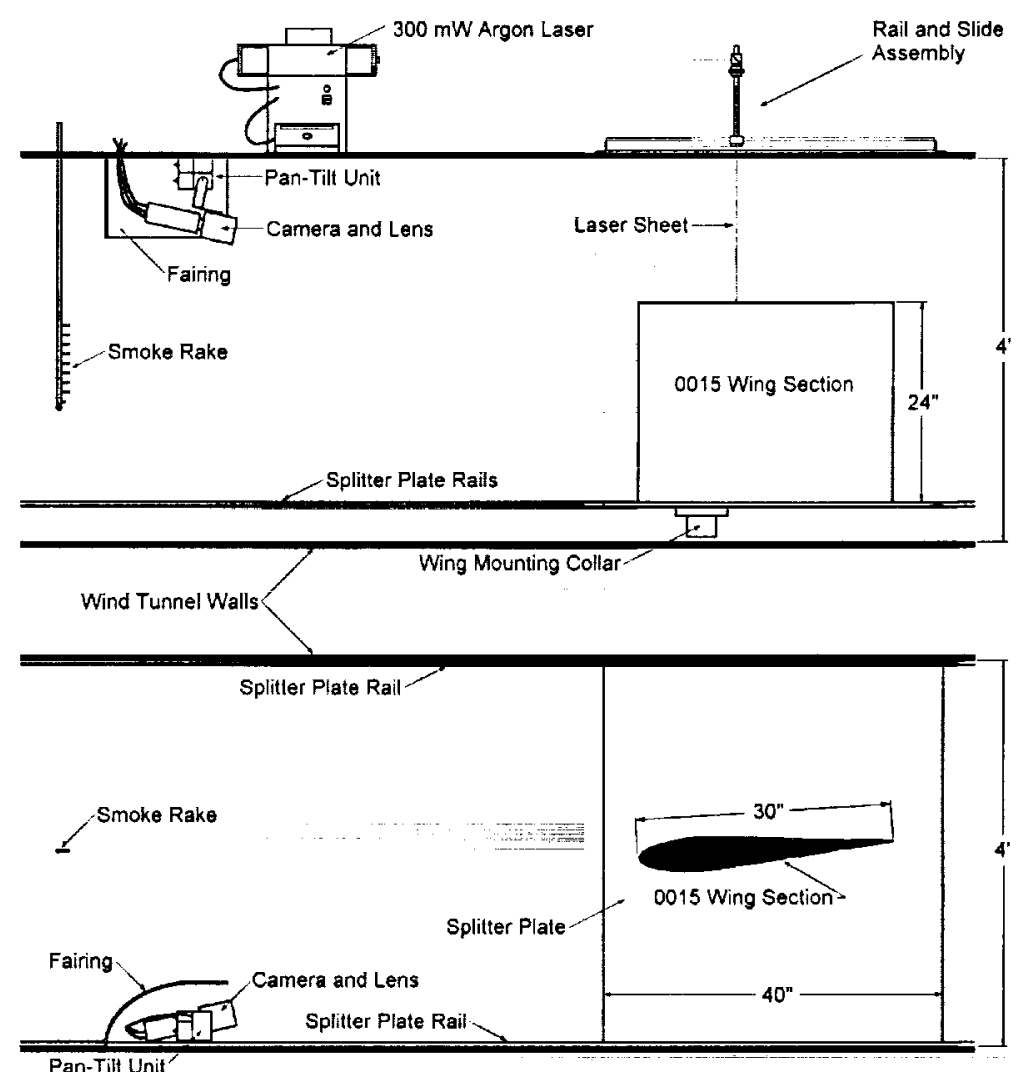

Figure 6. Flow visualization components in relation to the tunnel test section. 


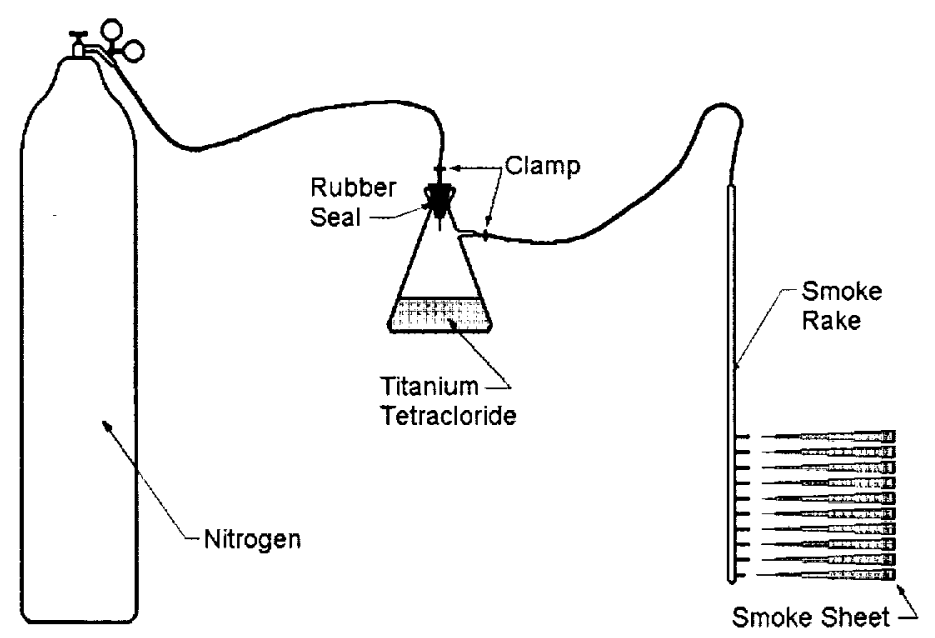

Figure 7. Flow seeding apparatus using titanium tetrachloride.

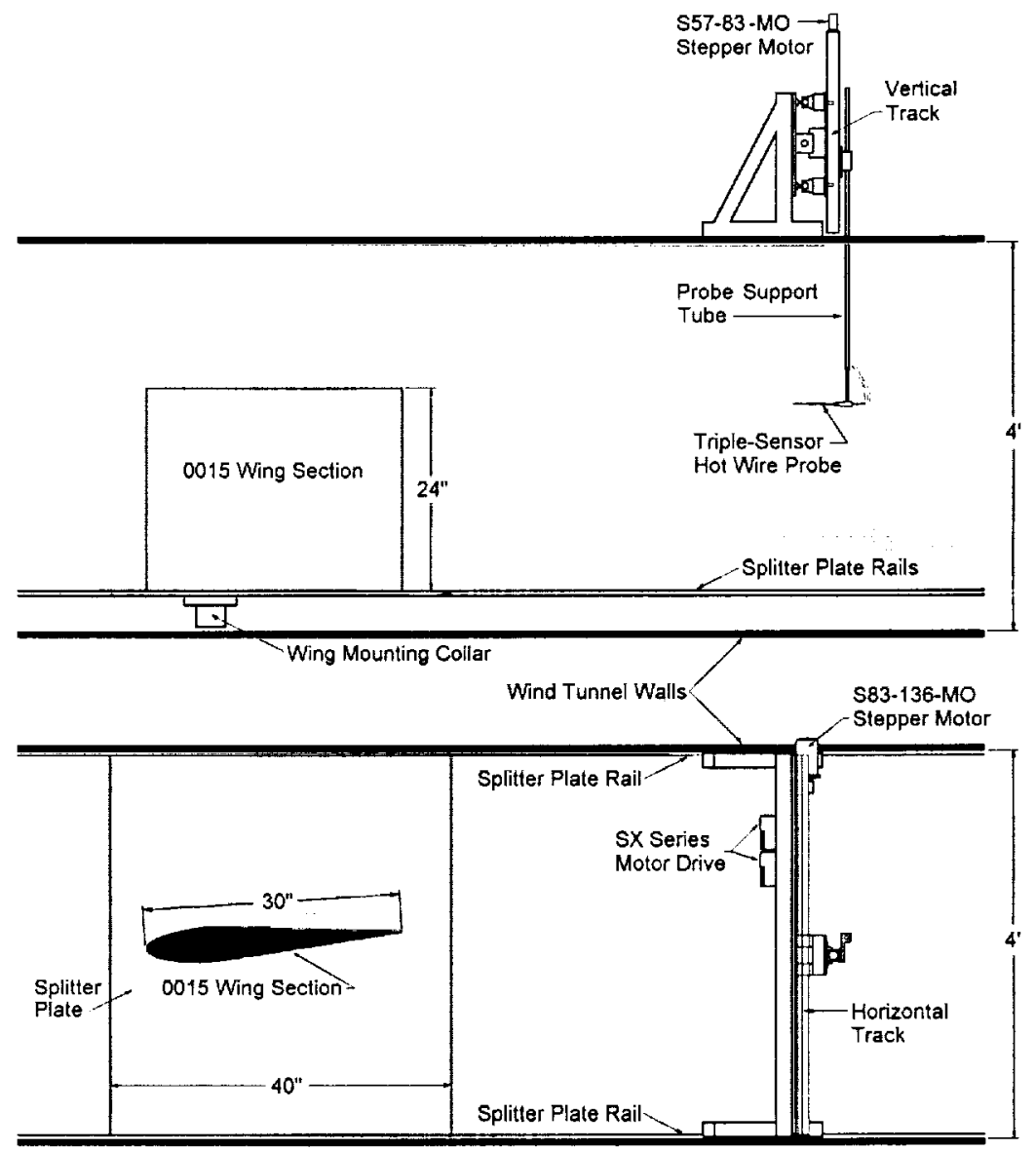

Figure 8 . Velocity measurement components in relation to the tunnel test section. 


\section{Three-Sensor Probe With No Common Prong} Model AVEP-3-102

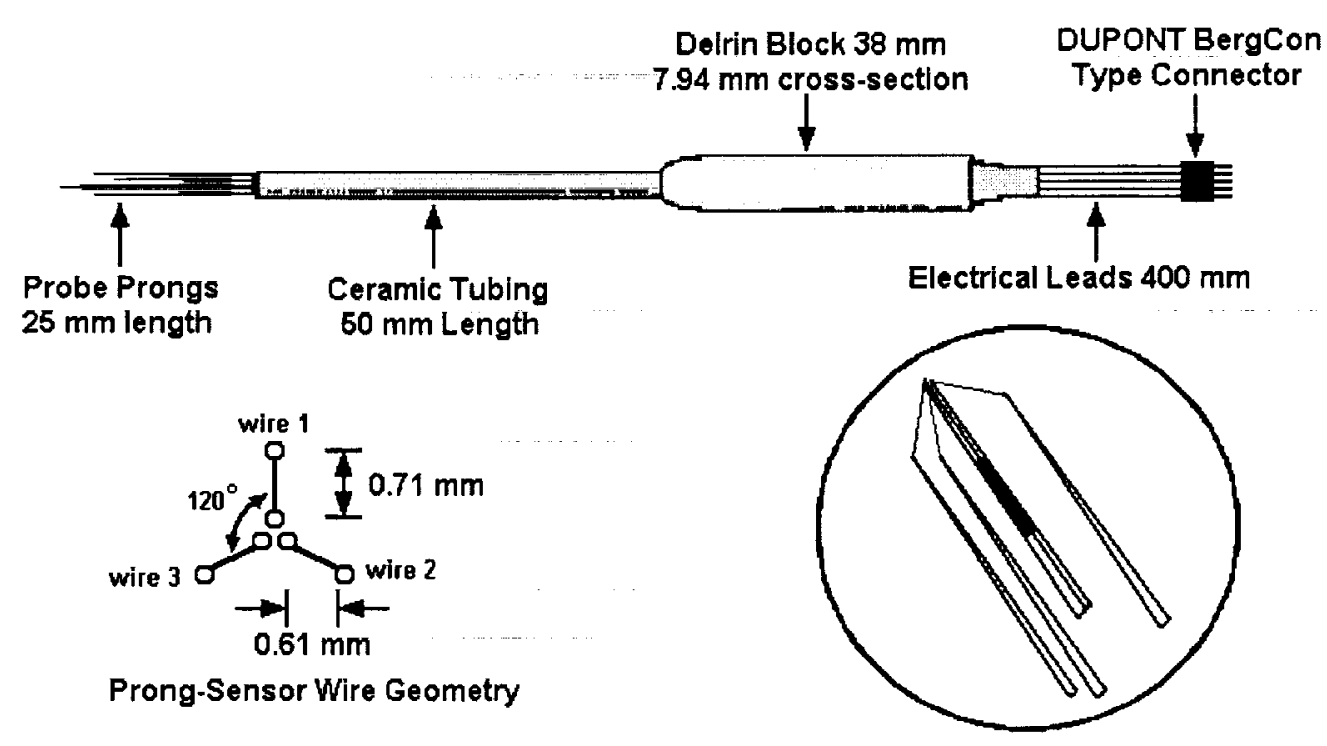

Figure 9. Auspex AVEP-3-102 triple-sensor hot wire probe.
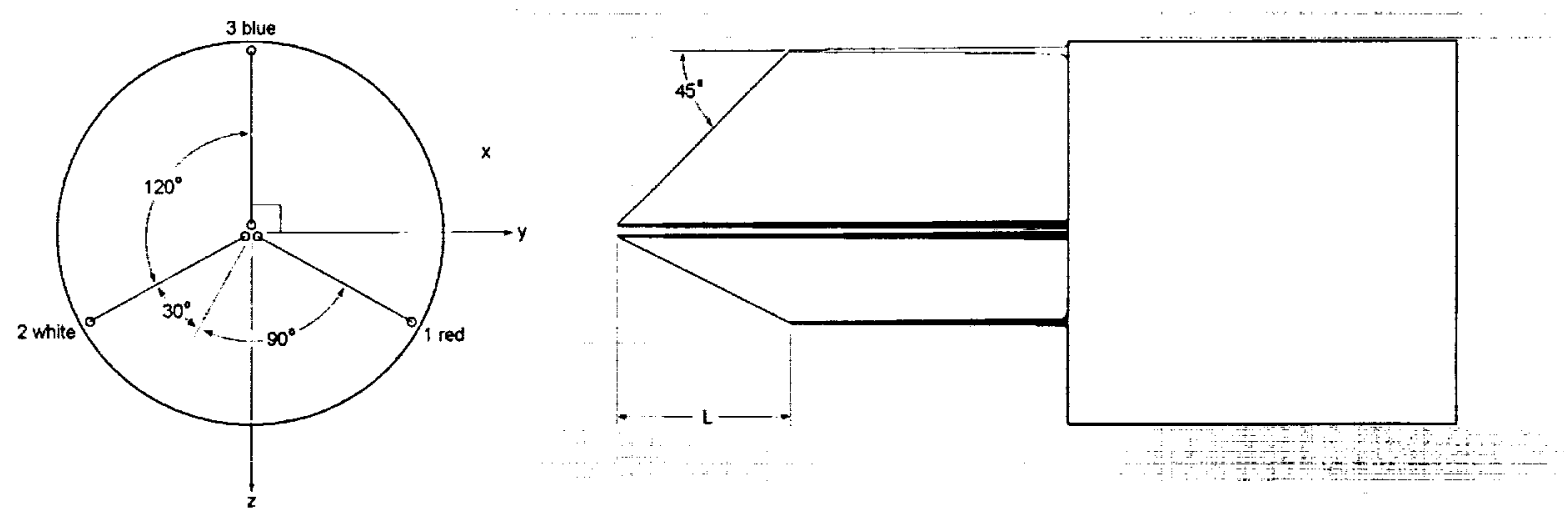

Figure 10. Sensor array geometry of the triple-sensor probe. 


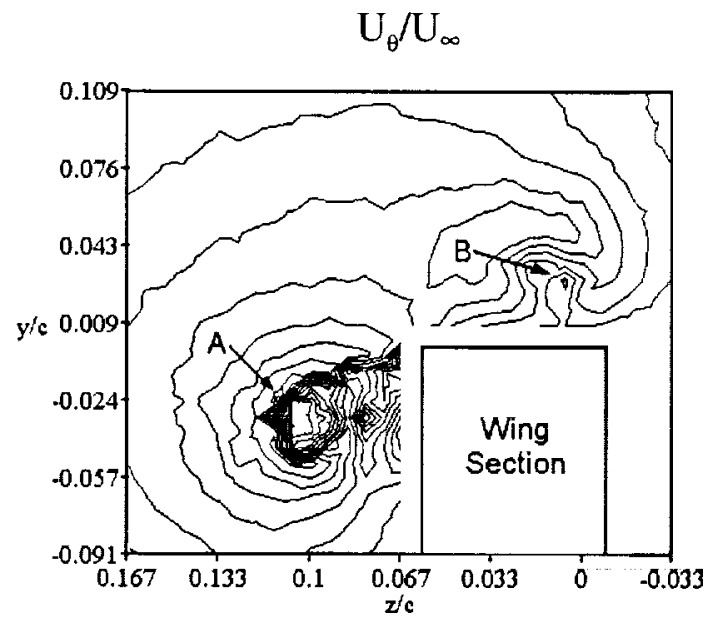

(a) $\Delta=0.05$

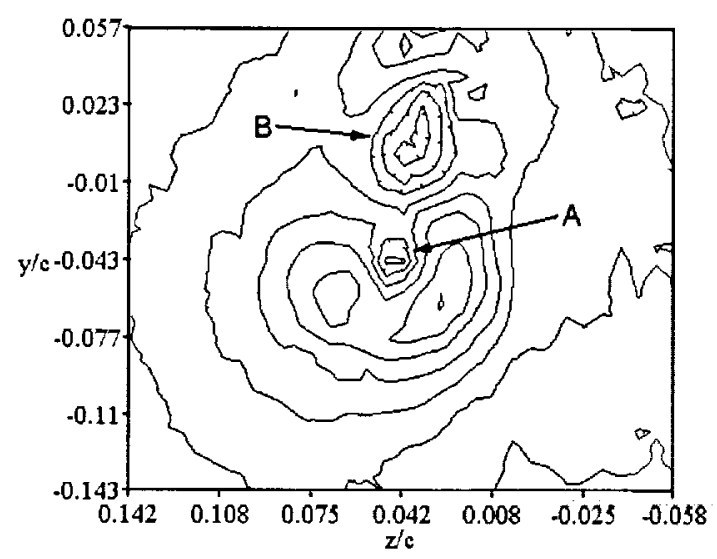

(c) $\Delta=0.05$

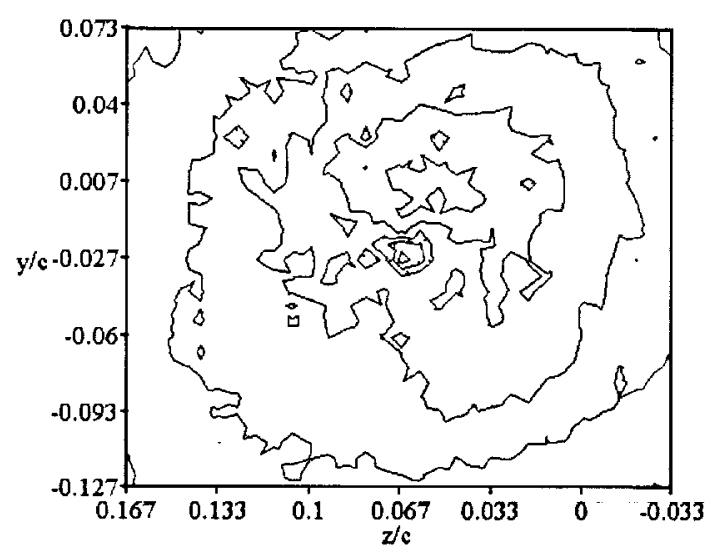

(e) $\Delta=0.05$

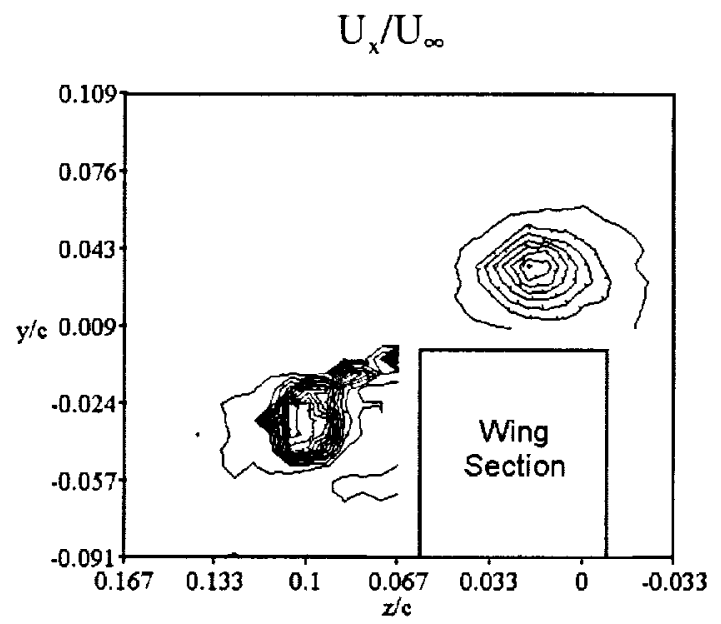

(b) $\Delta=0.10$

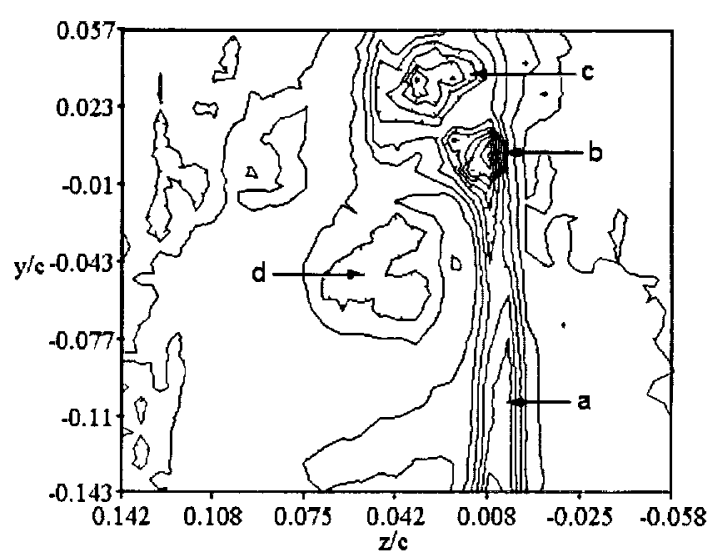

(d) $\Delta=0.05$

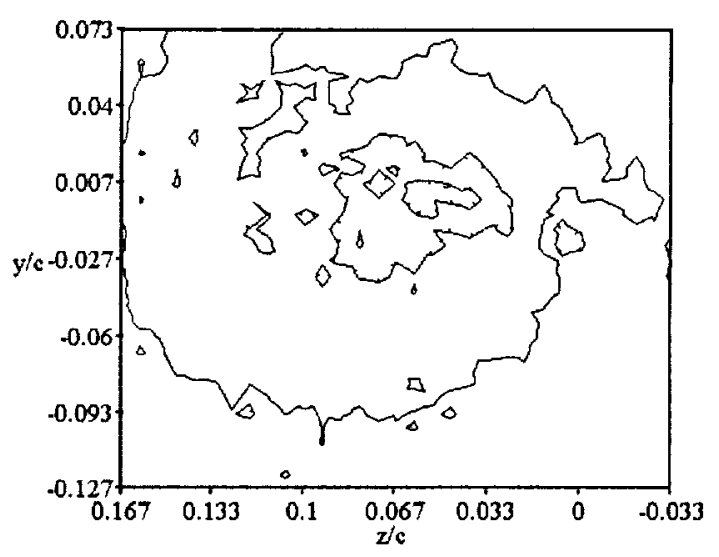

(f) $\Delta=0.05$

Figure 11 a-f. Tangential velocity contours (a, c, and e) and axial velocity contours (b, d, and f) for the flat end-cap configuration at $\alpha=4^{\circ}$. 


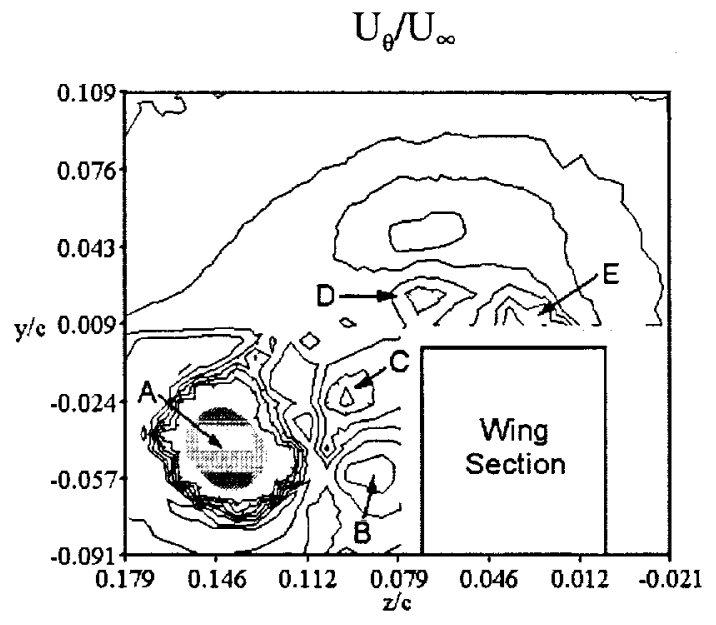

(a) $\Delta=0.10$

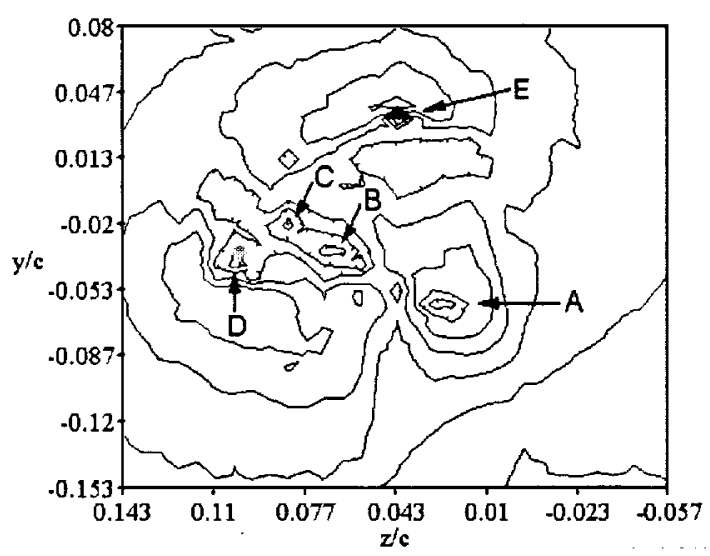

(c) $\Delta=0.10$

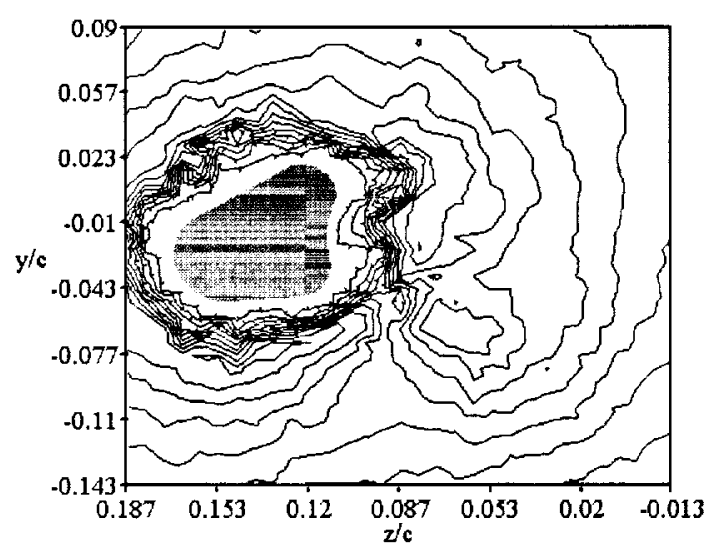

(e) $\Delta=0.05$

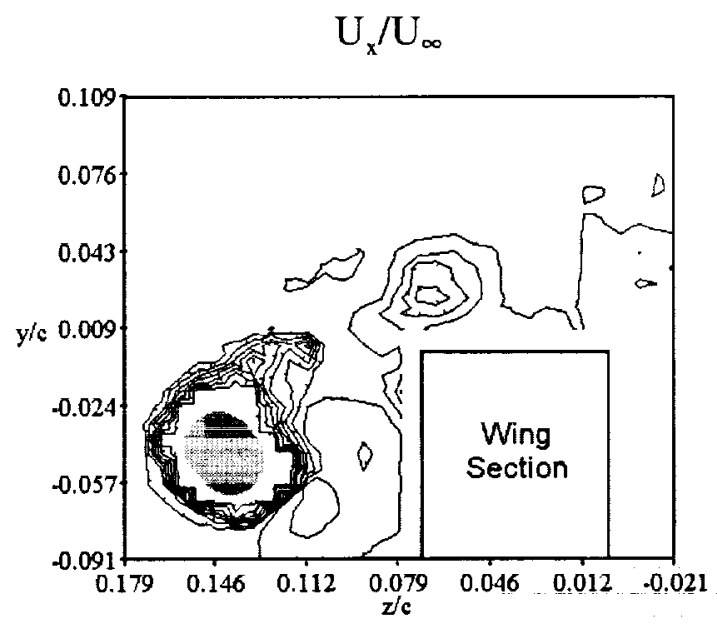

(b) $\Delta=0.15$

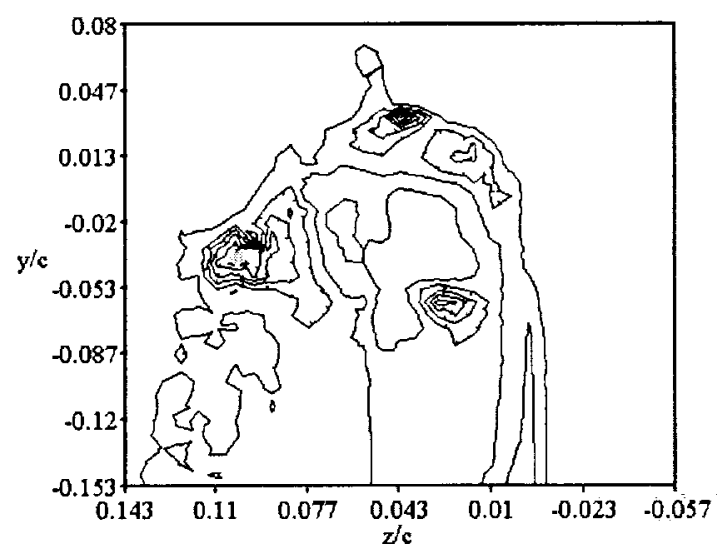

(d) $\Delta=0.15$

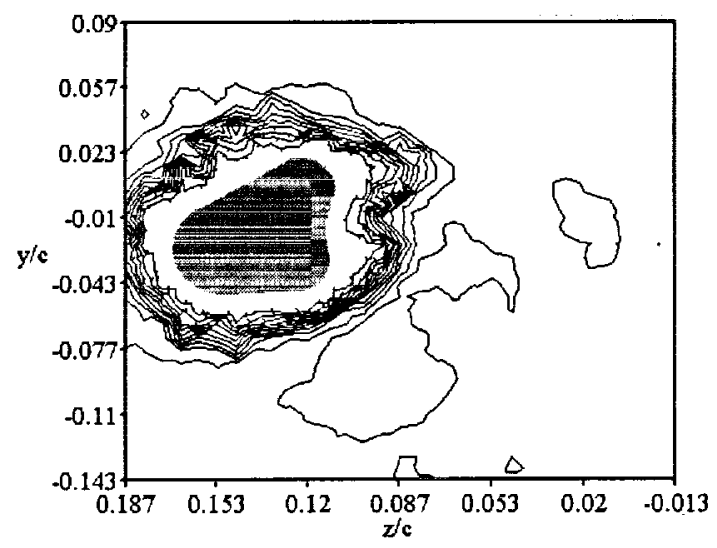

(f) $\Delta=0.10$

Figure 12 a-f. Tangential velocity contours (a, c, and e) and axial velocity contours $(b, d$, and $f$ ) for the flat end-cap configuration at $\alpha=8^{\circ}$. 
$\mathrm{U}_{\theta} / \mathrm{U}_{\infty}$

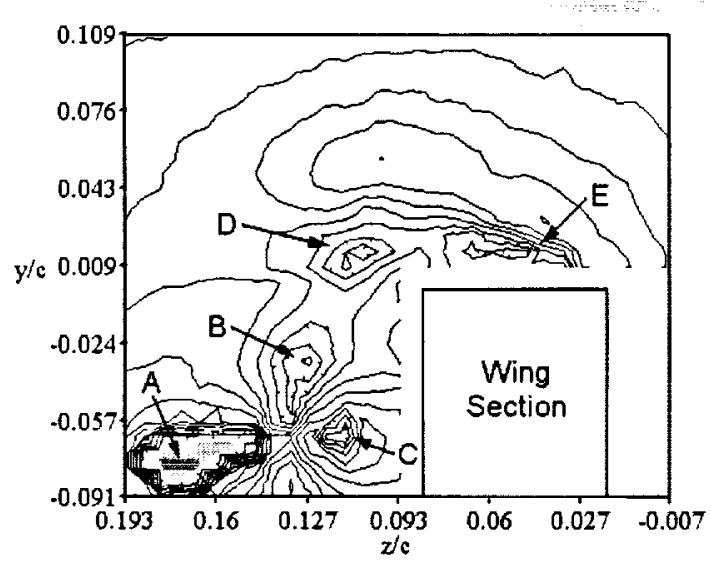

(a) $\Delta=0.10$

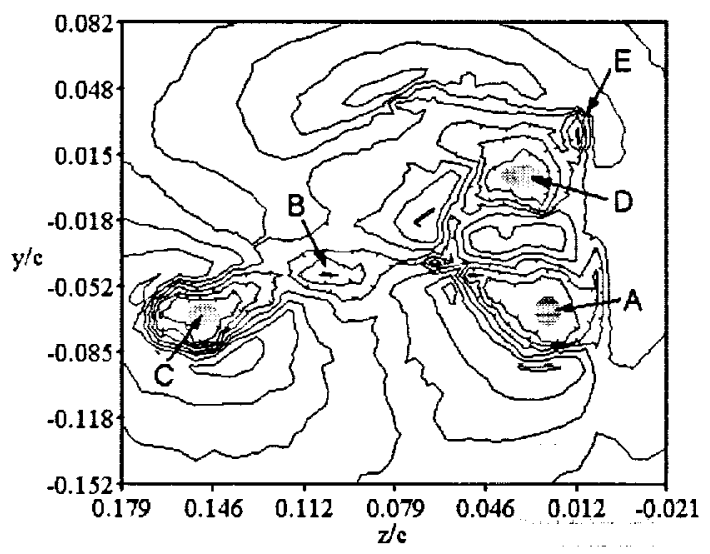

(c) $\Delta=0.10$

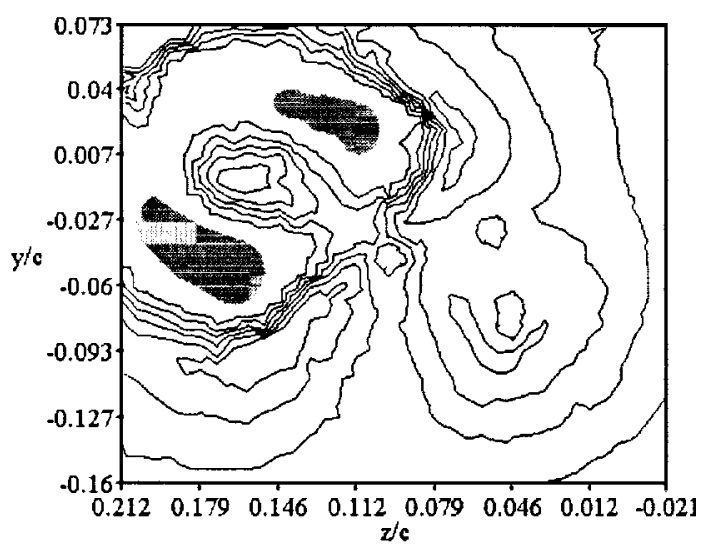

(e) $\Delta=0.10$
$\mathrm{U}_{\mathrm{x}} / \mathrm{U}_{\infty}$

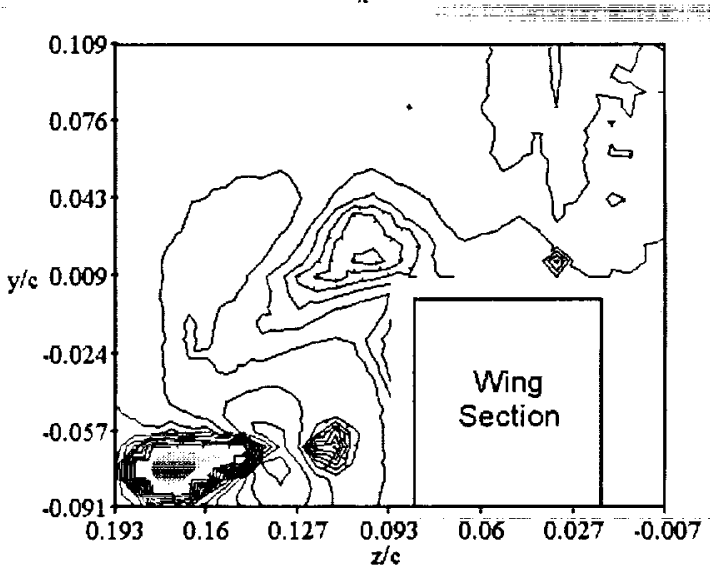

(b) $\Delta=0.15$

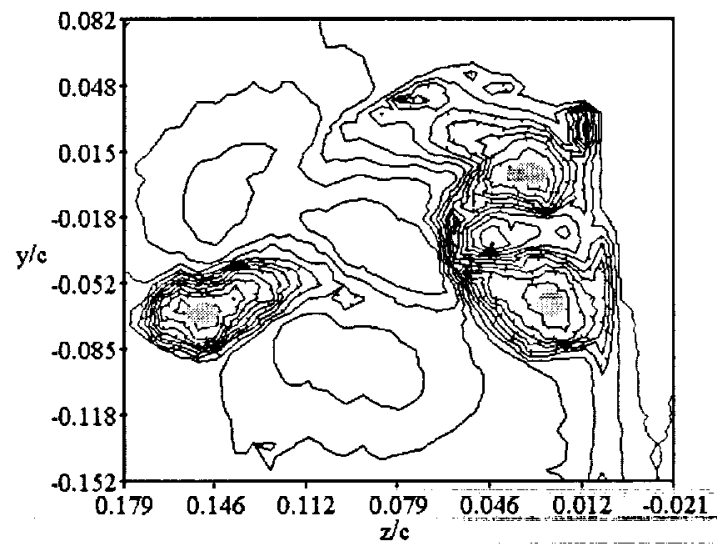

(d) $\Delta=0.15$

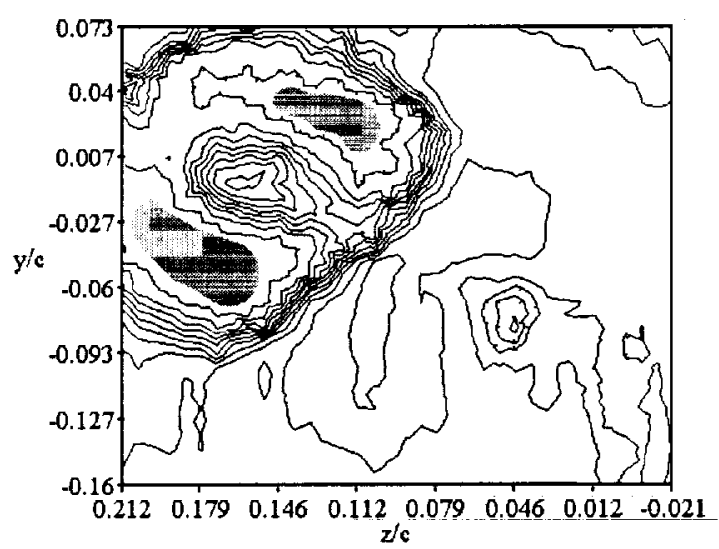

(f) $\Delta=0.15$

Figure 13 a-f. Tangential velocity contours (a, c, and e) and axial velocity contours (b, d, and $f$ ) for the flat end-cap configuration at $\alpha=12^{\circ}$. 


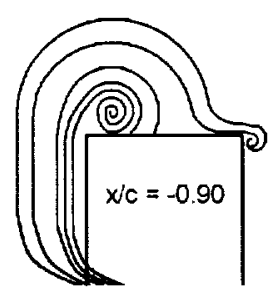

Front

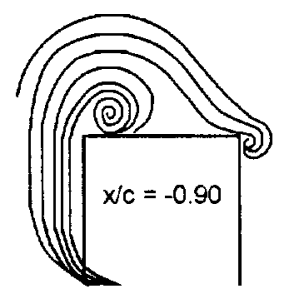

Front

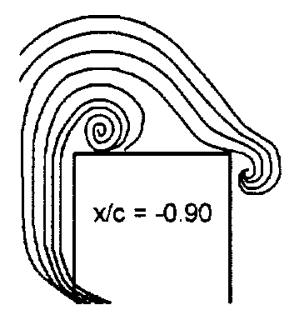

Front

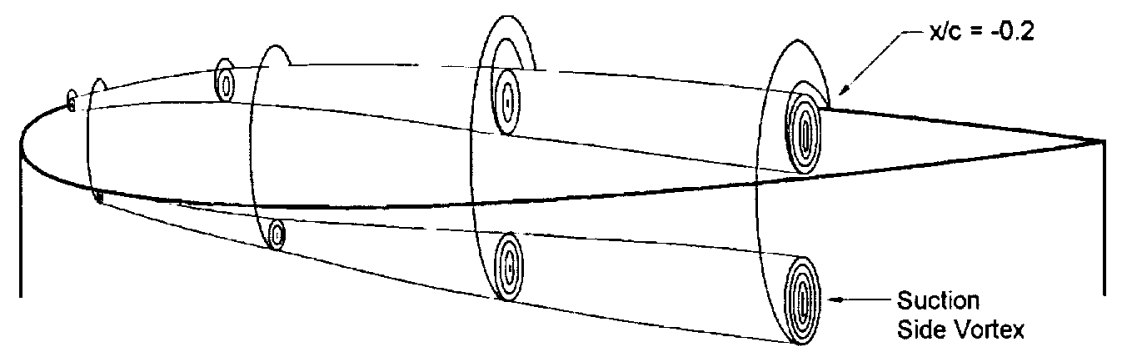

Top / Side

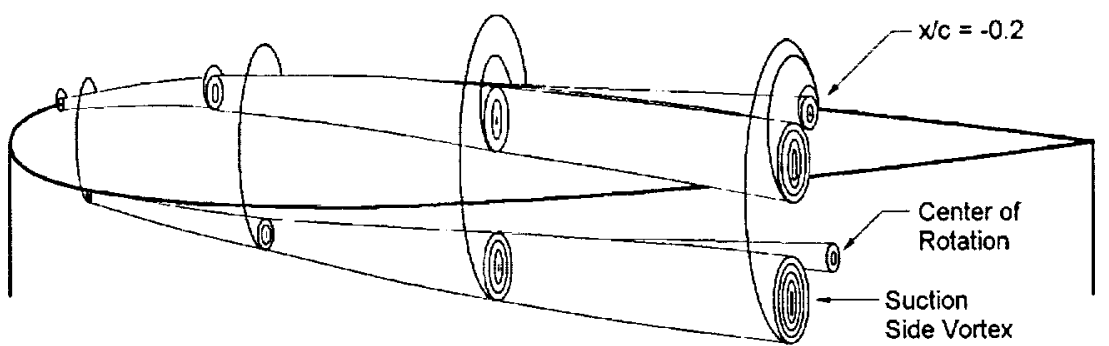

Top / Side

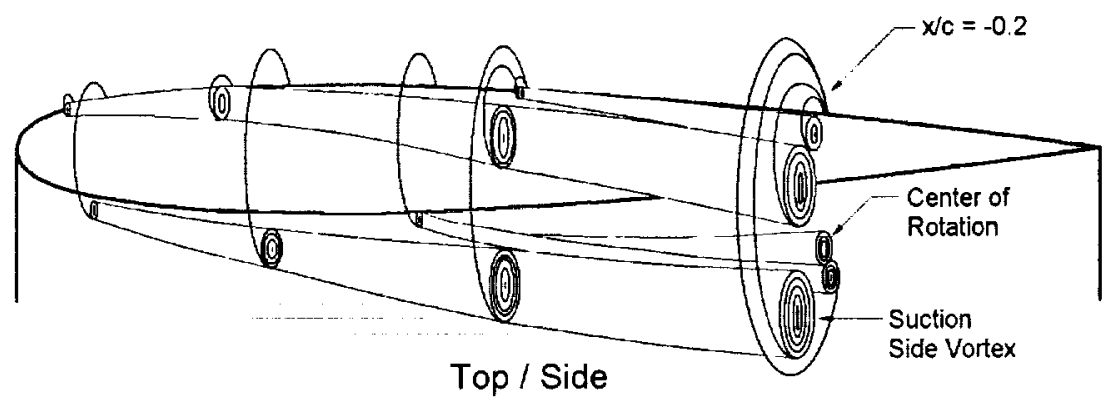

Figure 14 a-c. The initial formation and progression of the multiple vortices generated from the wing section fitted with a flat end-cap. 


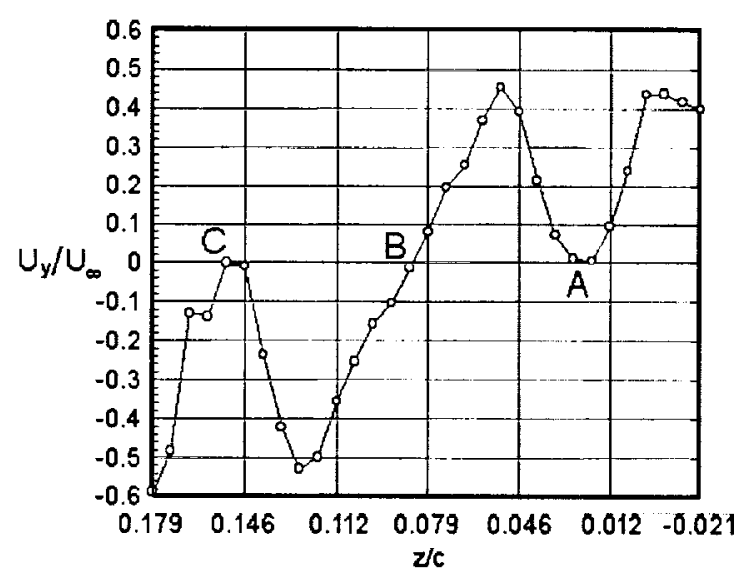

(a)

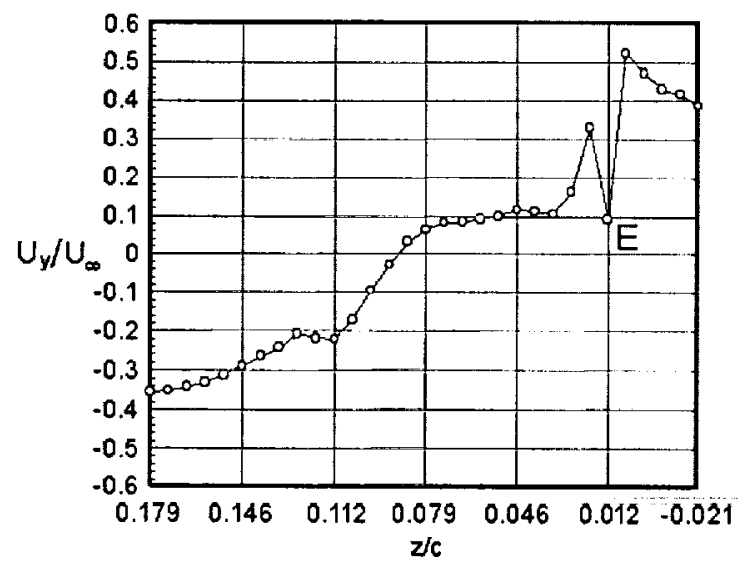

(c)

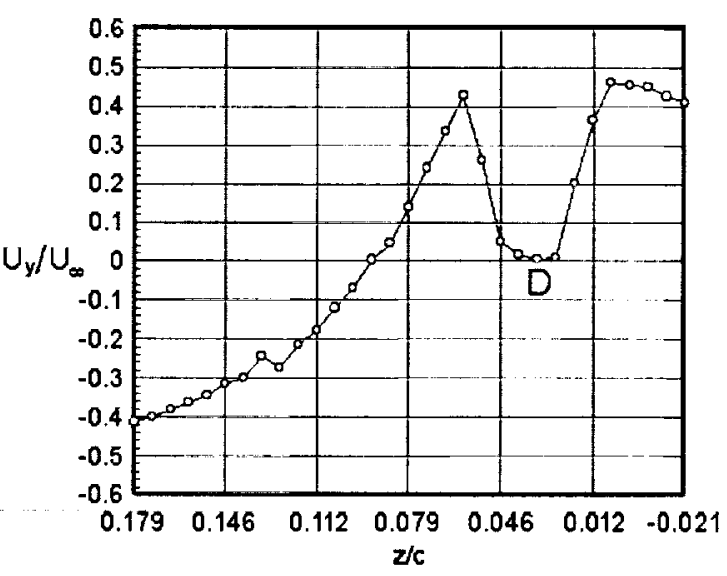

(b)

Figure 15 a-c. Normalized vertical velocity profiles for the vortices shown in Figure 12c.

(a), $y / c=-0.072 ;(b), y / c=0.002 ;(c), y / c=0.022$. 


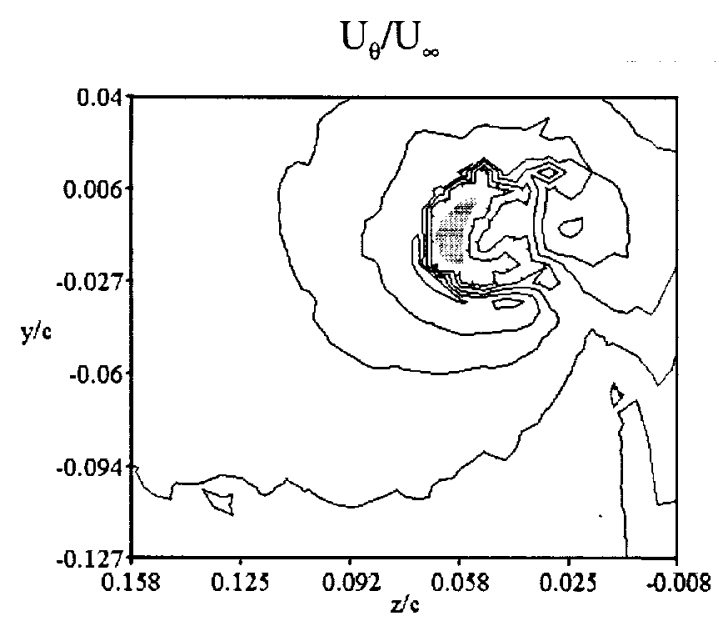

(a) $\Delta=0.10$

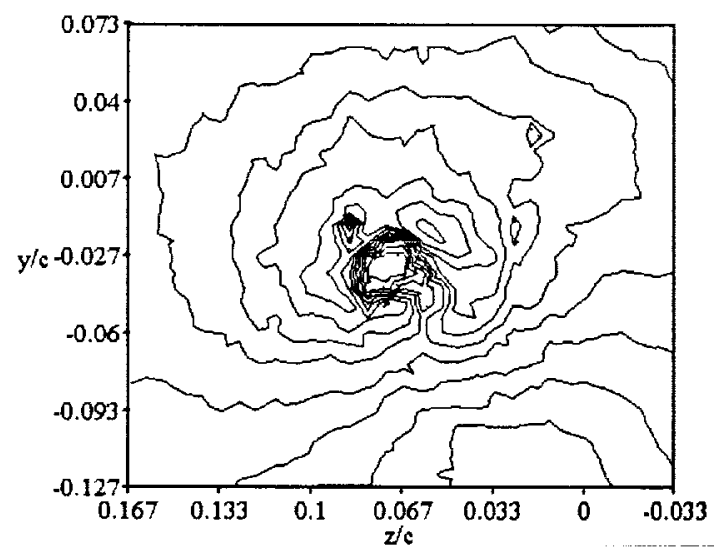

(c) $\Delta=0.05$

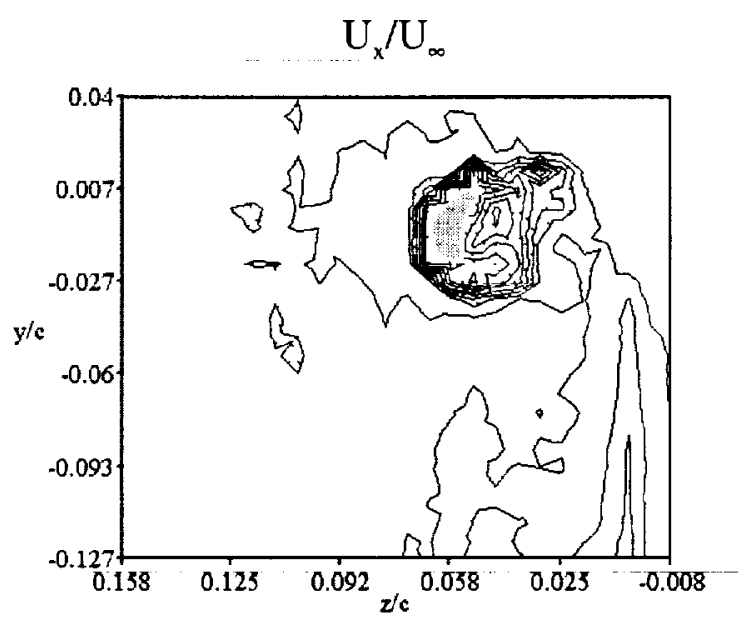

(b) $\Delta=0.15$

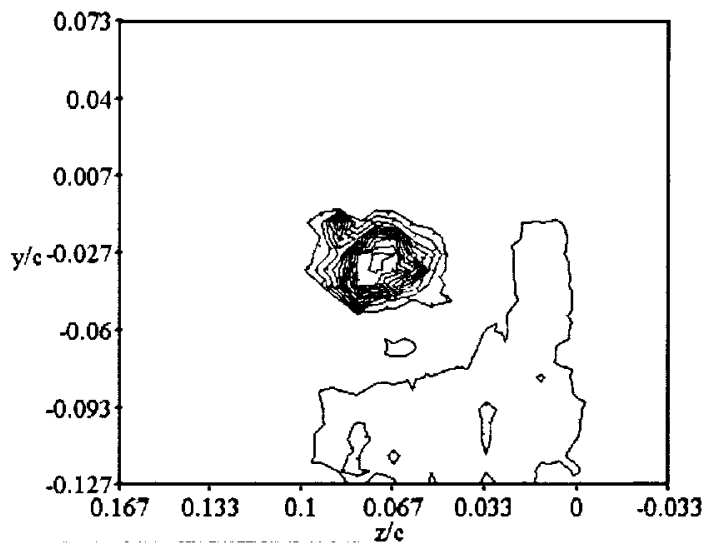

(d) $\Delta=0.10$

Figure 16 a-d. Tangential velocity contours ( $a$ and $c$ ) and axial velocity contours (b and d) for the round end-cap configuration at $\alpha=4^{\circ}$. 


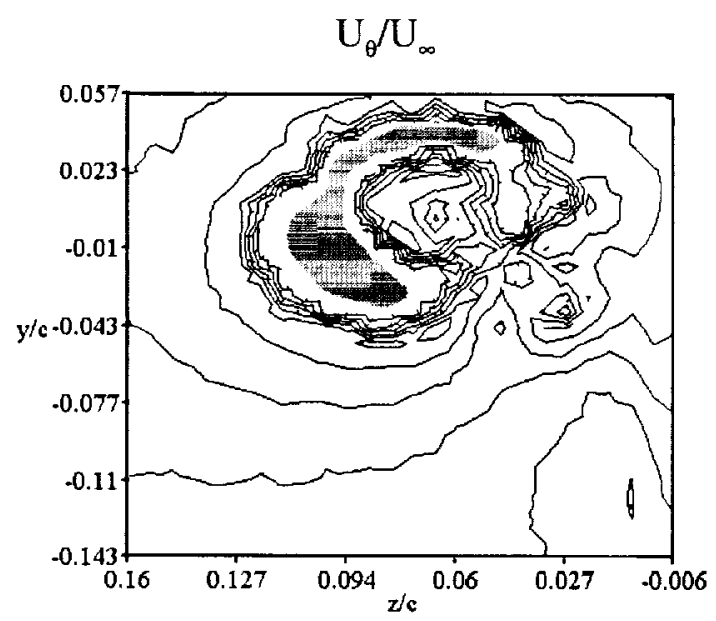

(a) $\Delta=0.10$

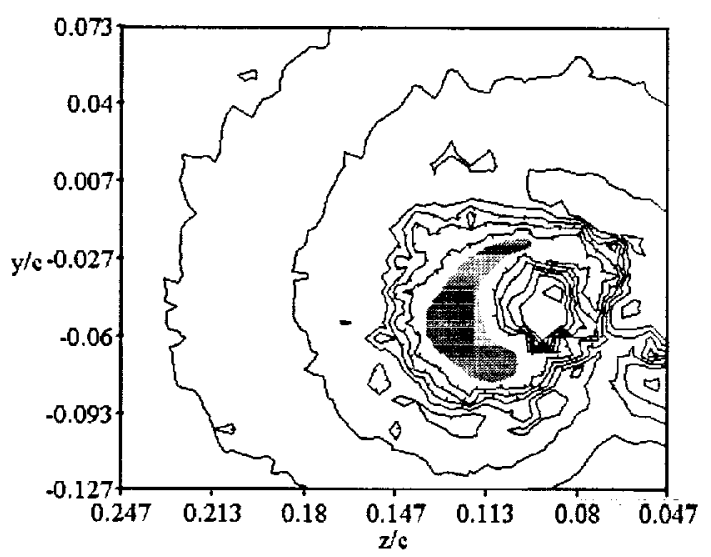

(c) $\Delta=0.10$

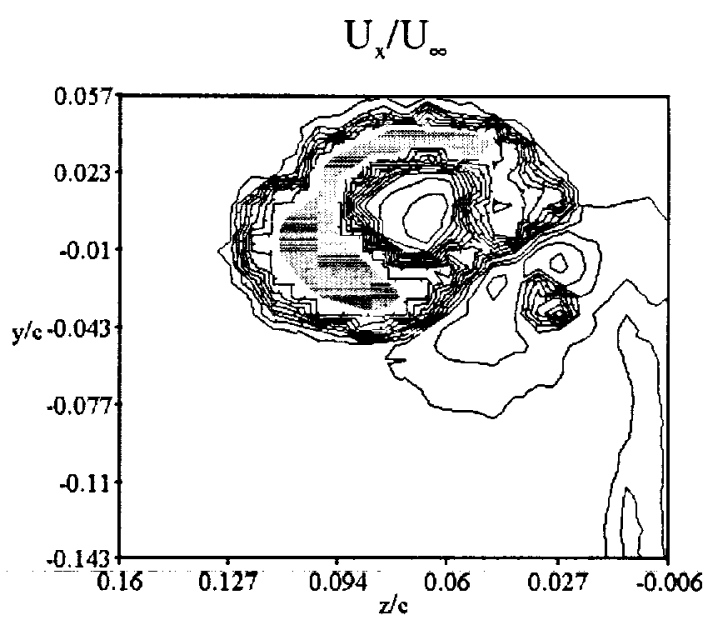

(b) $\Delta=0.15$

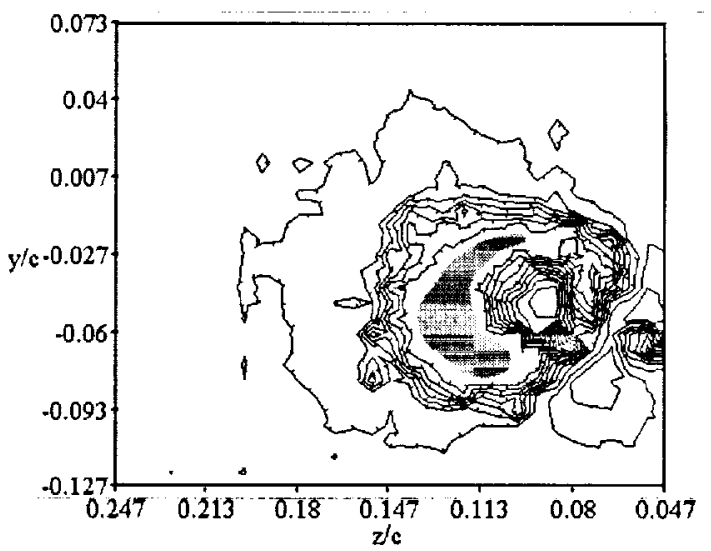

(d) $\Delta=0.15$

Figure 17 a-d. Tangential velocity contours ( $a$ and $c$ ) and axial velocity contours (b and $d$ ) for the round end-cap configuration at $\alpha=8^{\circ}$. 


$$
\mathrm{U}_{\theta} / \mathrm{U}_{\infty}
$$

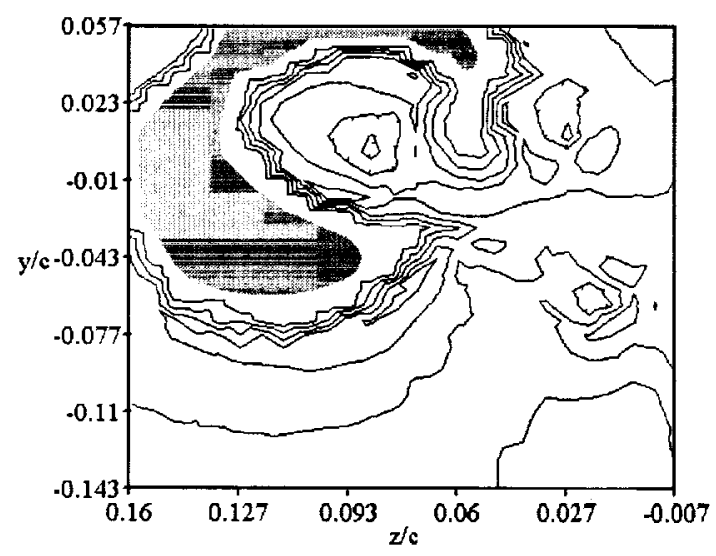

(a) $\Delta=0.15$

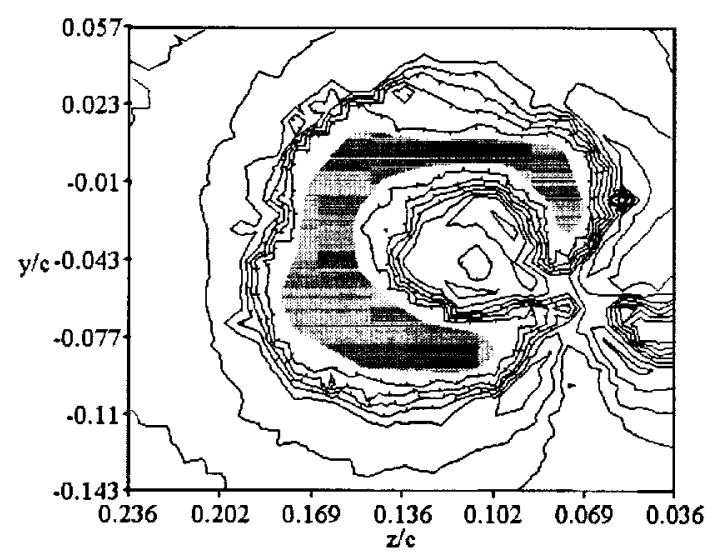

(c) $\Delta=0.10$

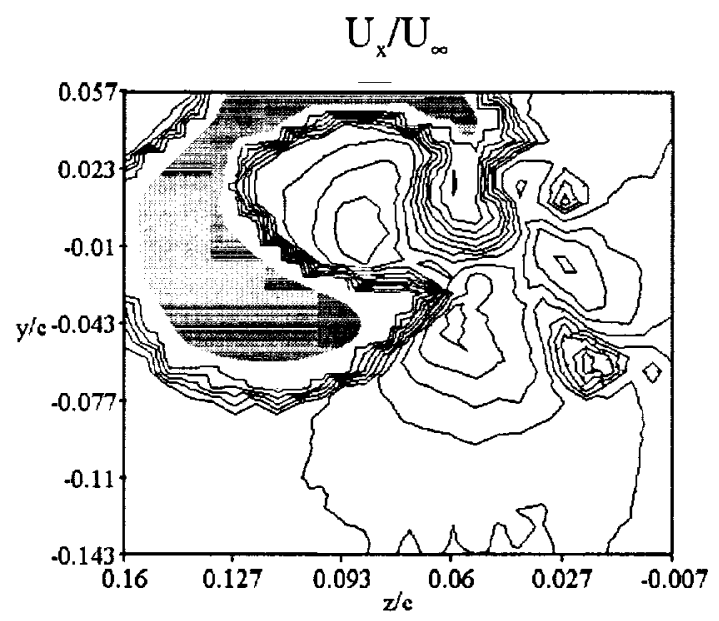

(b) $\Delta=0.20$

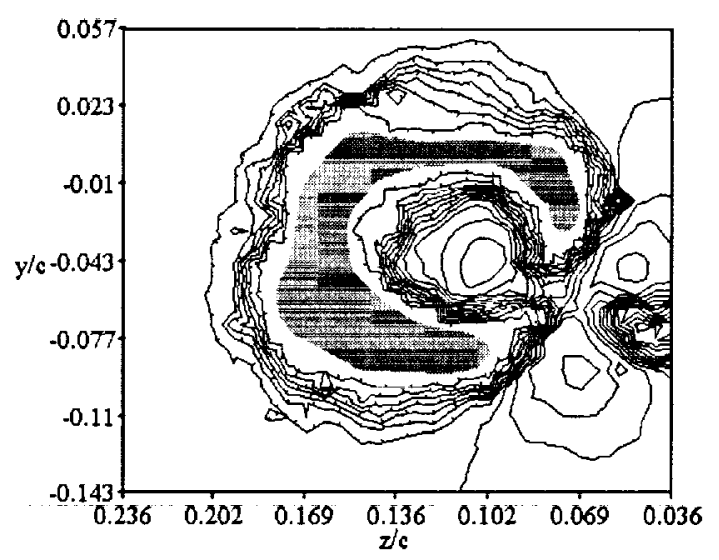

(d) $\Delta=0.15$

Figure $18 \mathrm{a}-\mathrm{d}$. Tangential velocity contours ( $\mathrm{a}$ and $\mathrm{c}$ ) and axial velocity contours ( $\mathrm{b}$ and $\mathrm{d}$ ) for the round end-cap configuration at $\alpha=12^{\circ}$. 


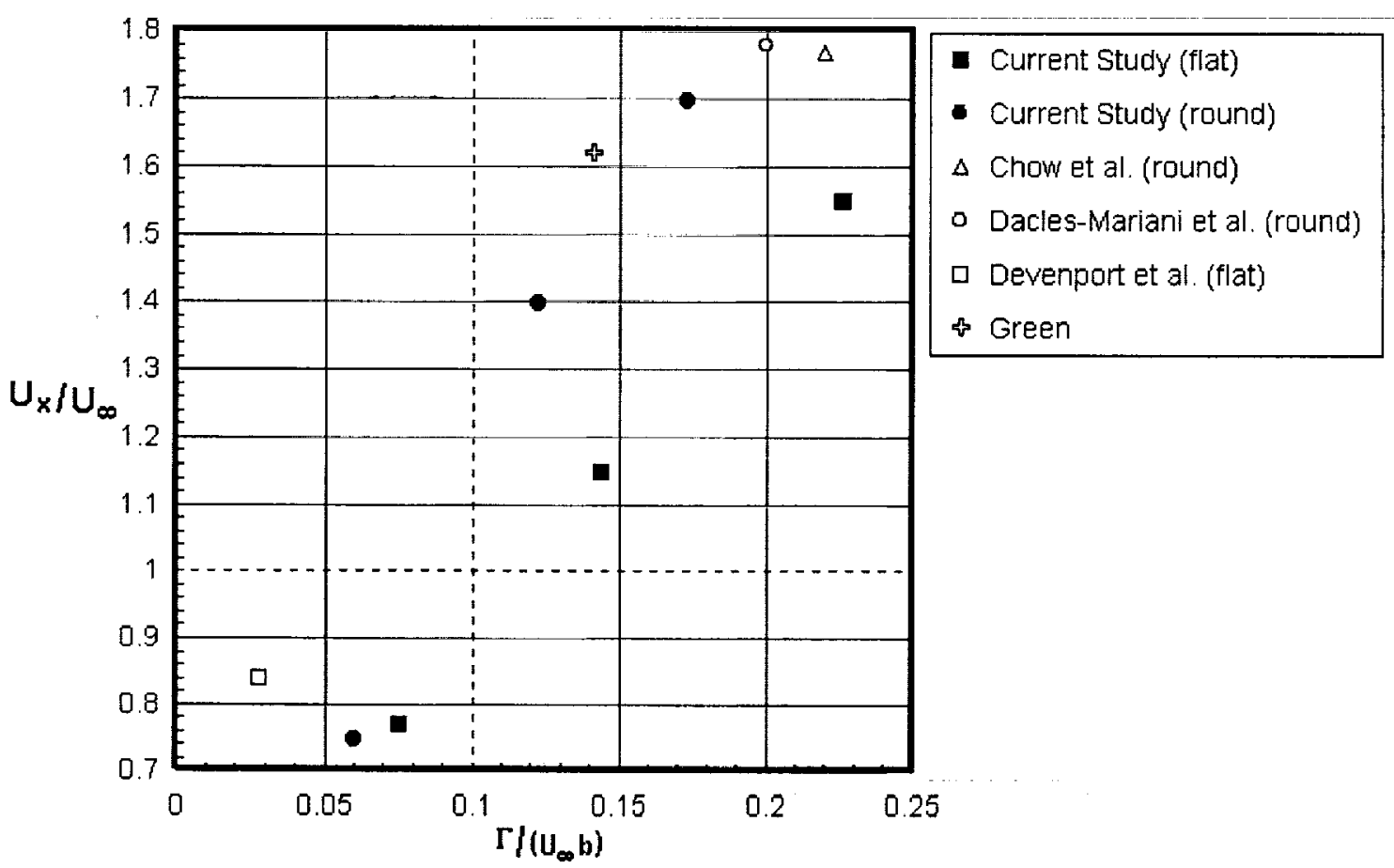

Figure 19. A comparison of the normalized axial velocity with the loading parameter. 


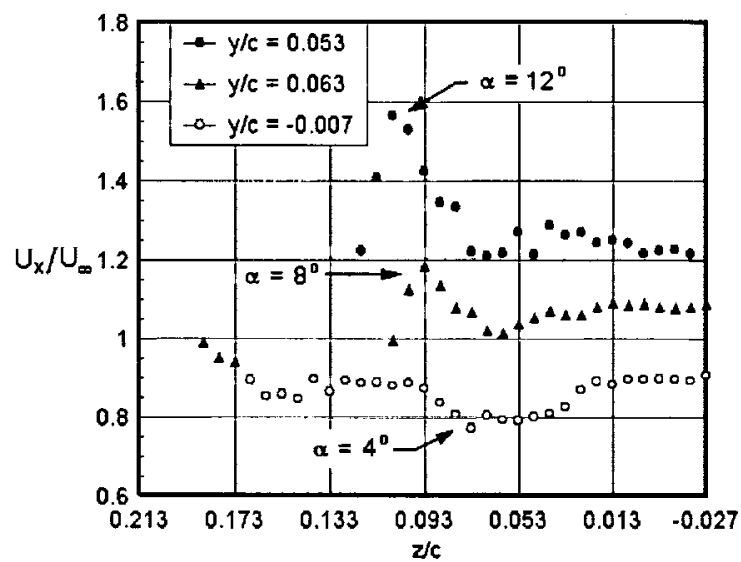

(a) Flat end-cap, $x / c=1.0$

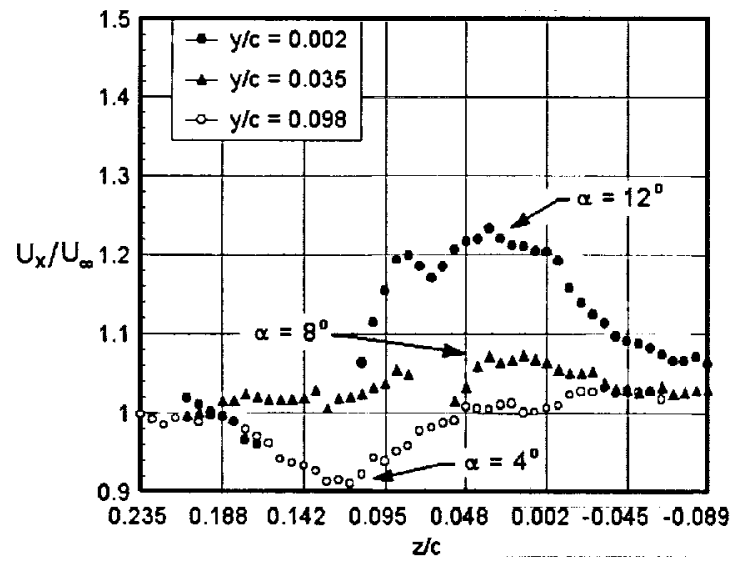

(c) Flat end-cap, $\mathrm{x} / \mathrm{c}=2.0$

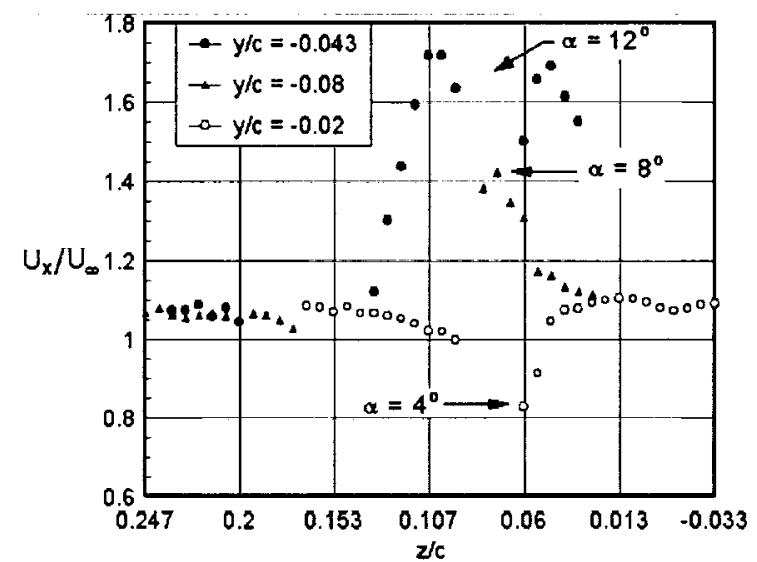

(b) round end-cap, $x / c=1.0$

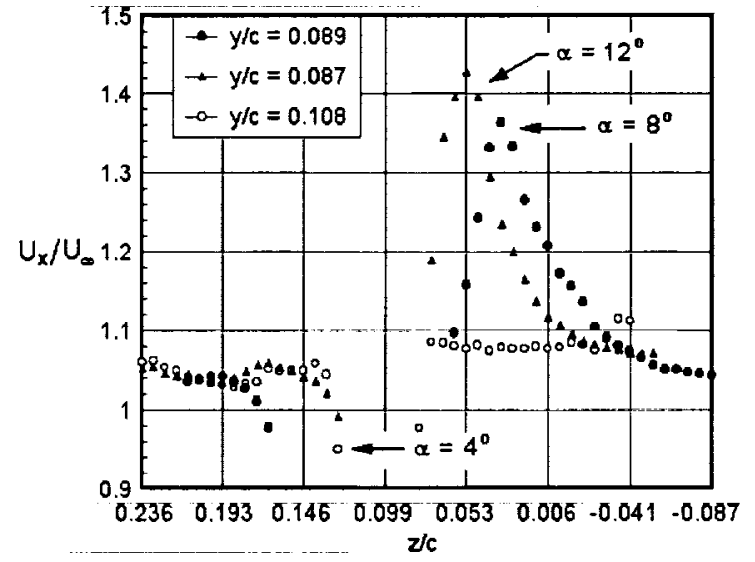

(d) round end-cap, $\mathrm{x} / \mathrm{c}=2.0$

Figure 20 a-d. Axial velocity profiles for the flat and round end-cap configurations at $\mathrm{x} / \mathrm{c}=1.0$ and $\mathrm{x} / \mathrm{c}=2.0$. 


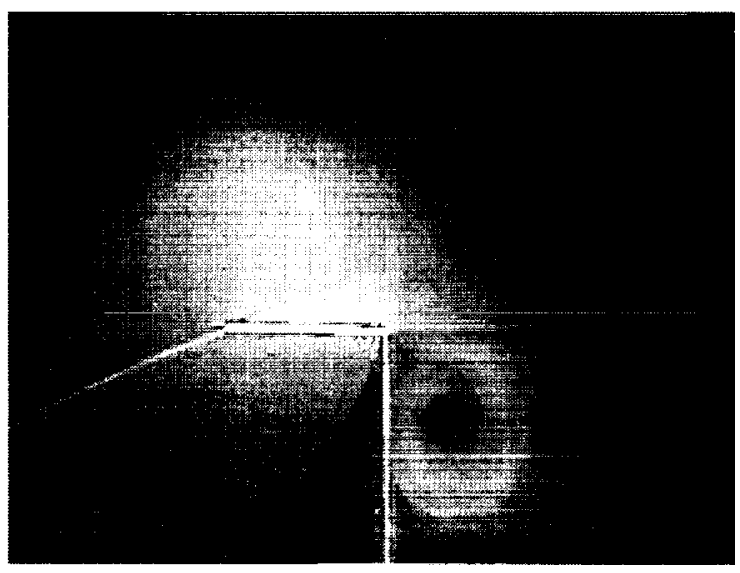

(a)

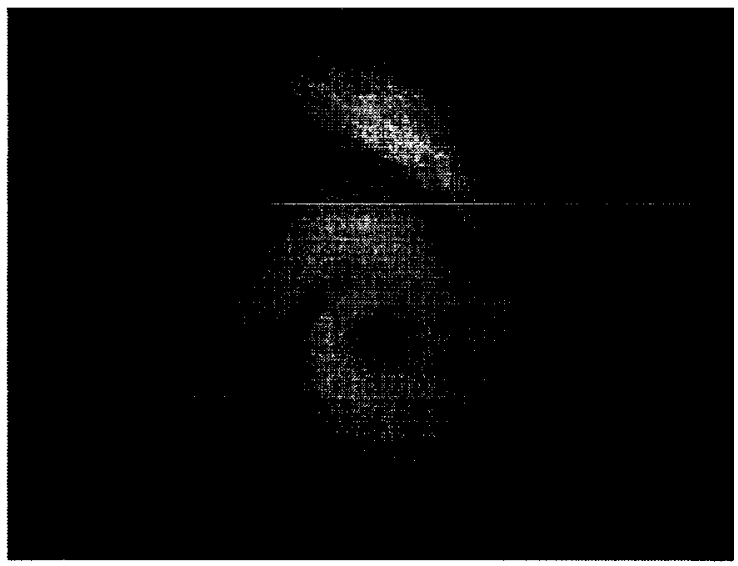

(c)

Figure 21 a-c. Flow visualization images for the flat end-cap at $\alpha=4^{\circ}$. (a), $x / c=-0.20$; (b), $\mathrm{x} / \mathrm{c}=0.05 ;(\mathrm{c}) \mathrm{x} / \mathrm{c}=1.0$.

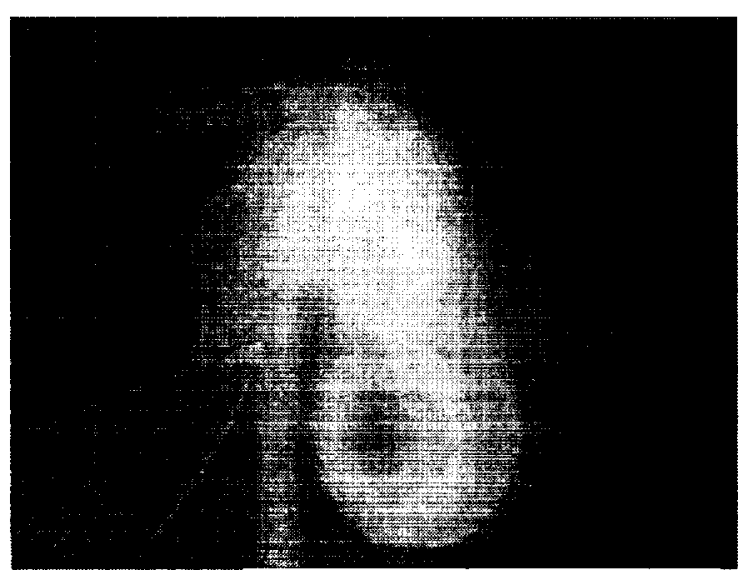

(b) 


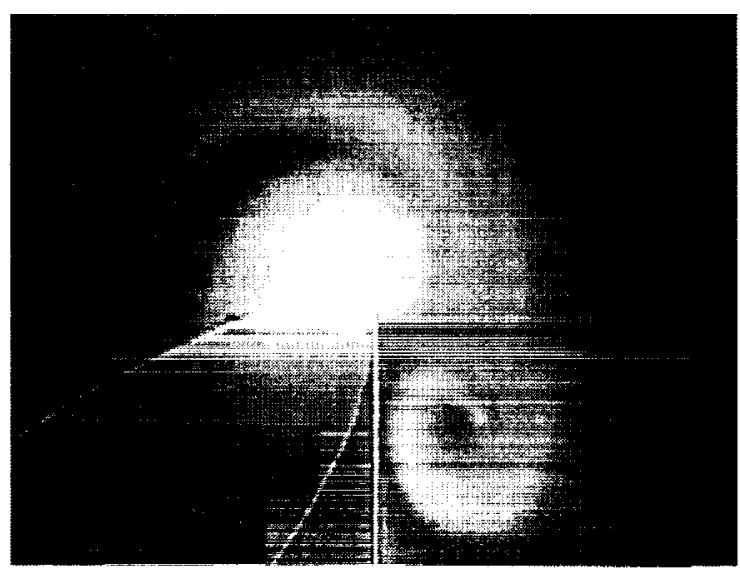

(a)

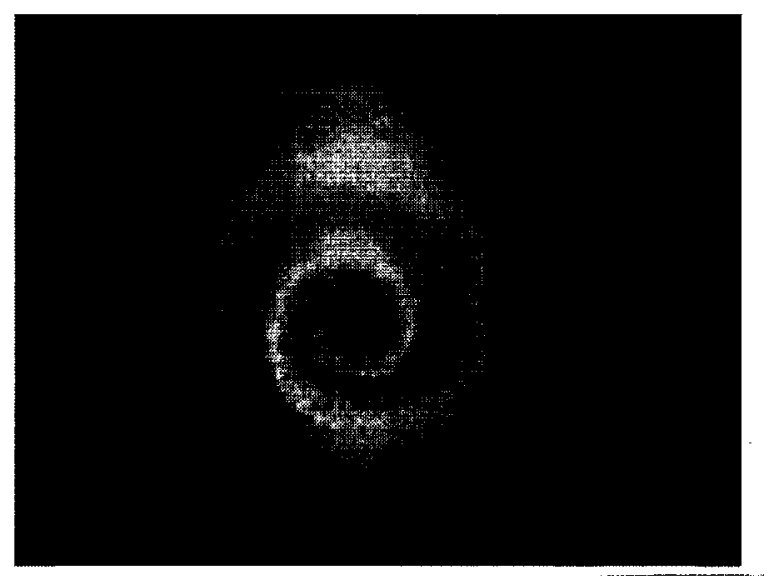

(c)

Figure $22 \mathrm{a}$-c. Flow visualization images for the flat end-cap at $\alpha=8^{\circ}$. (a), $x / c=-0.20$; (b), $\mathrm{x} / \mathrm{c}=0.05 ;(\mathrm{c}) \mathrm{x} / \mathrm{c}=1.0$.

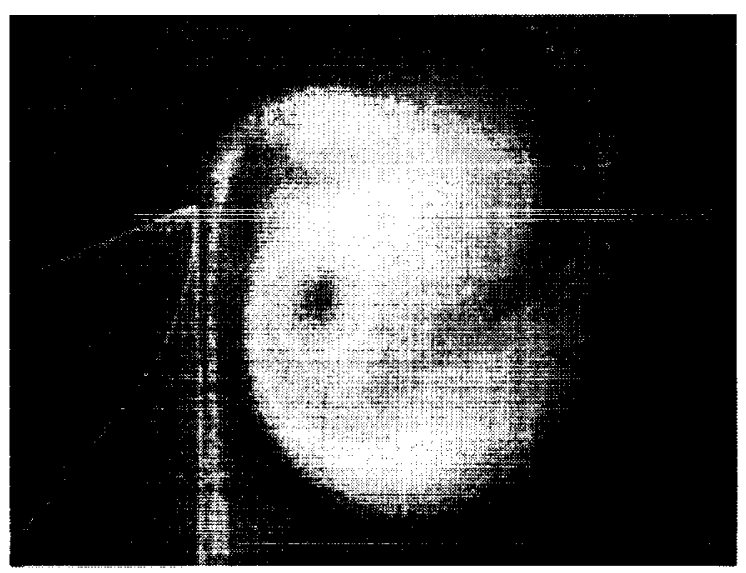

(b) 


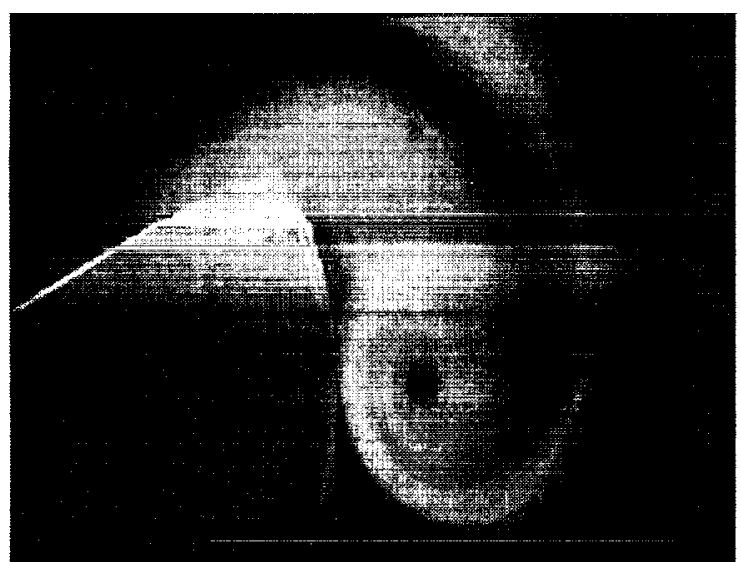

(a)

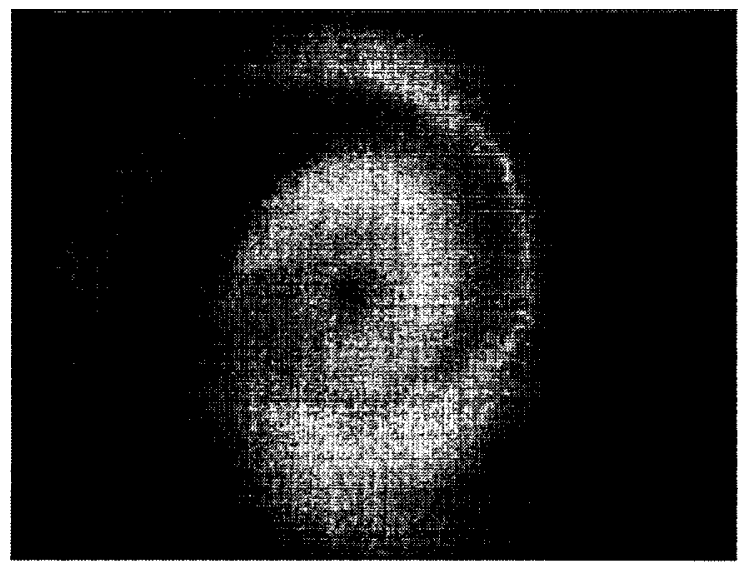

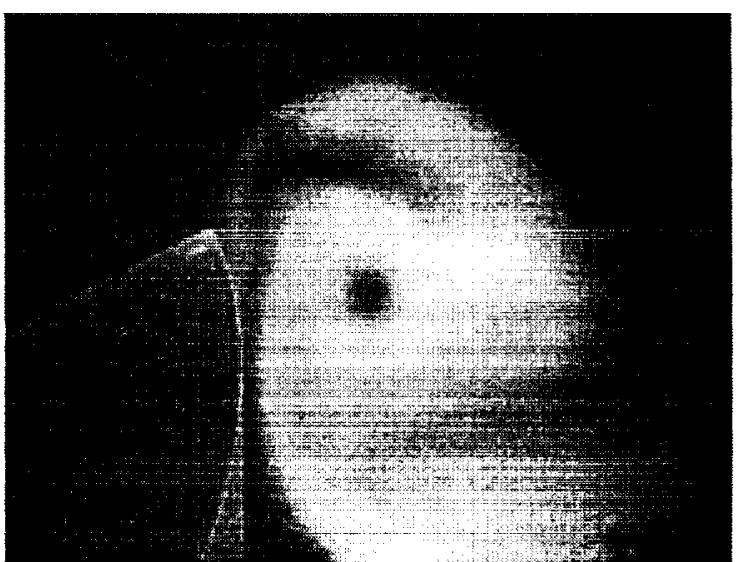

(b)

(c)

Figure 23 a-c. Flow visualization images for the flat end-cap at $\alpha=12^{\circ}$. (a), $x / c=-0.20$; (b), $\mathrm{x} / \mathrm{c}=0.05 ;(\mathrm{c}) \mathrm{x} / \mathrm{c}=1.0$. 


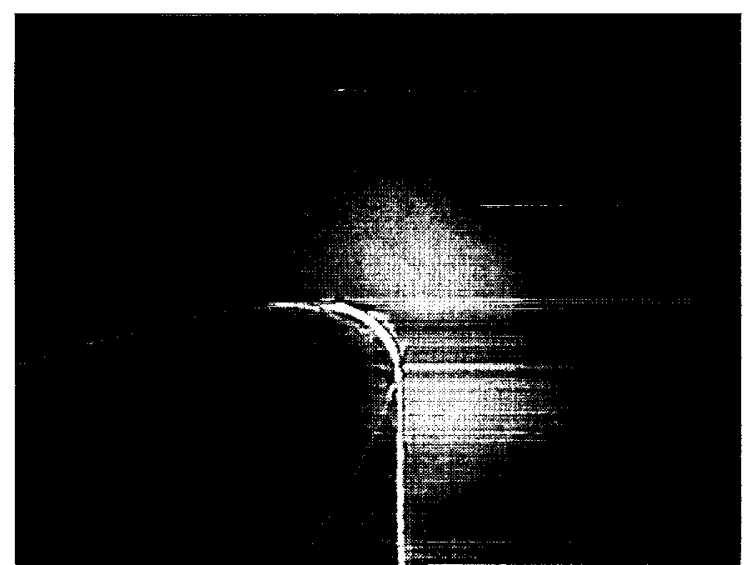

(a)

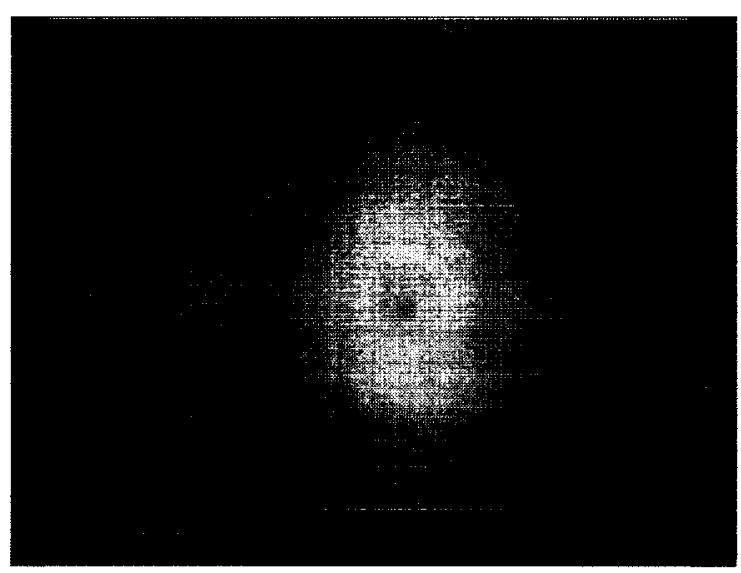

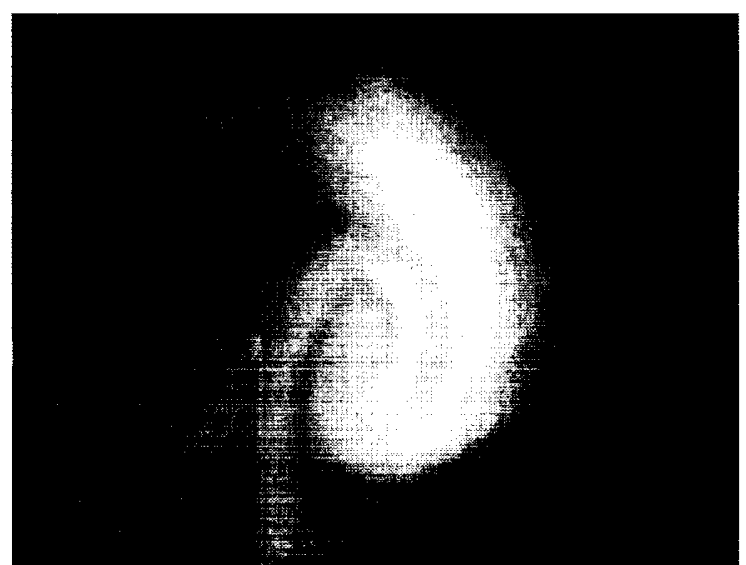

(b)

(c)

Figure 24 a-c. Flow visualization images for the round end-cap at $\alpha=4^{\circ}$. (a), $x / c=-0.15$; (b), $x / c=0.05 ;(c) x / c=1.0$. 


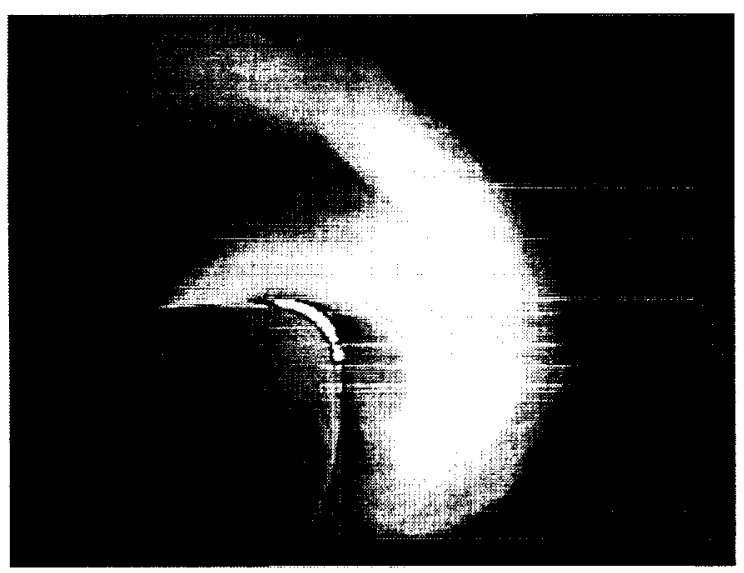

(a)

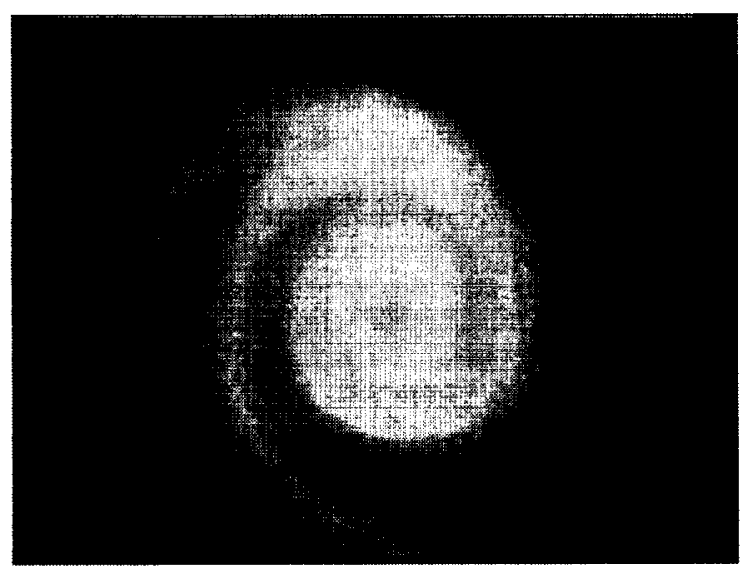

(c)

Figure 25 a-c. Flow visualization images for the round end-cap at $\alpha=8^{\circ}$. (a), $x / c=-0.15$; (b), $x / c=0.05 ;$ (c) $x / c=1.0$.

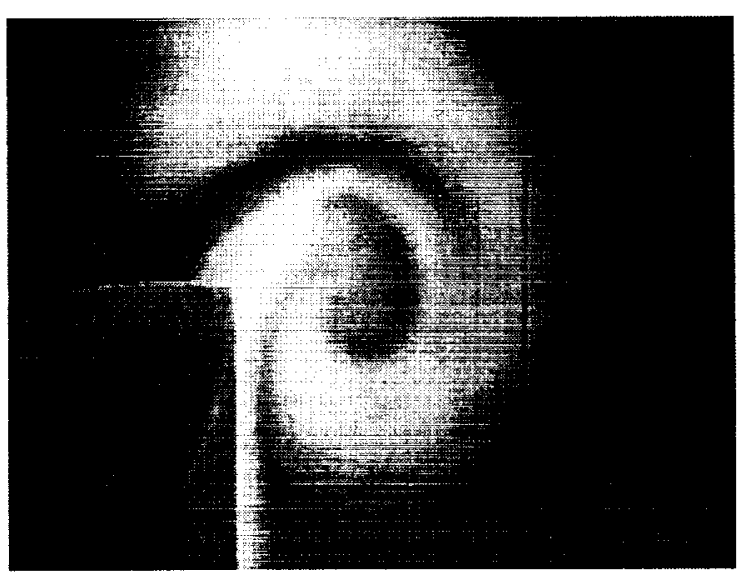

(b) 


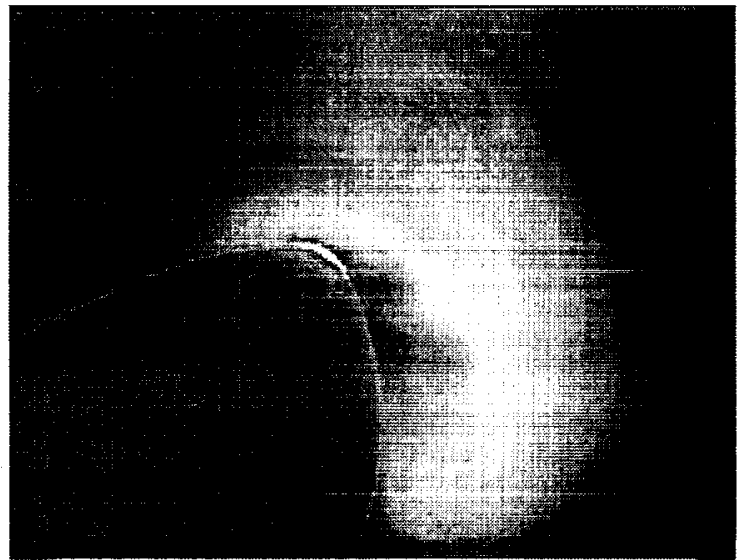

(a)

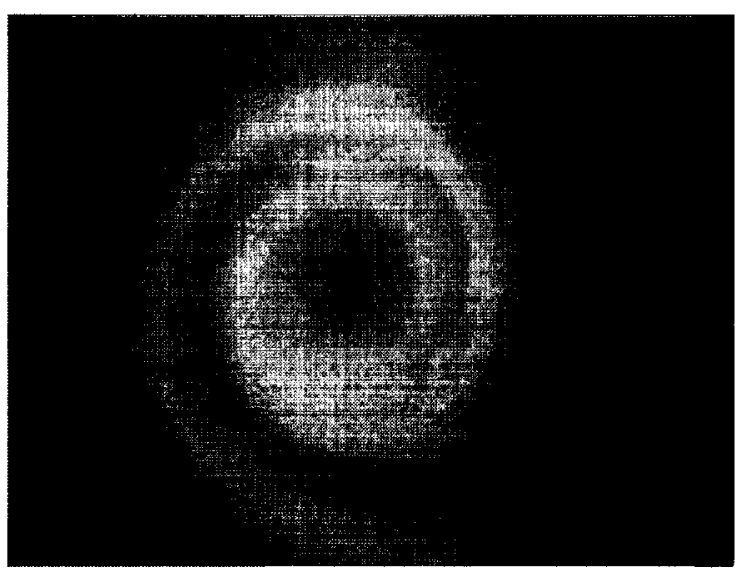

(c)

Figure 26 a-c. Flow visualization images for the round end-cap at $\alpha=12^{\circ}$. (a), $x / c=-0.15$; (b), $\mathrm{x} / \mathrm{c}=0.05 ;$ (c) $\mathrm{x} / \mathrm{c}=1.0$.

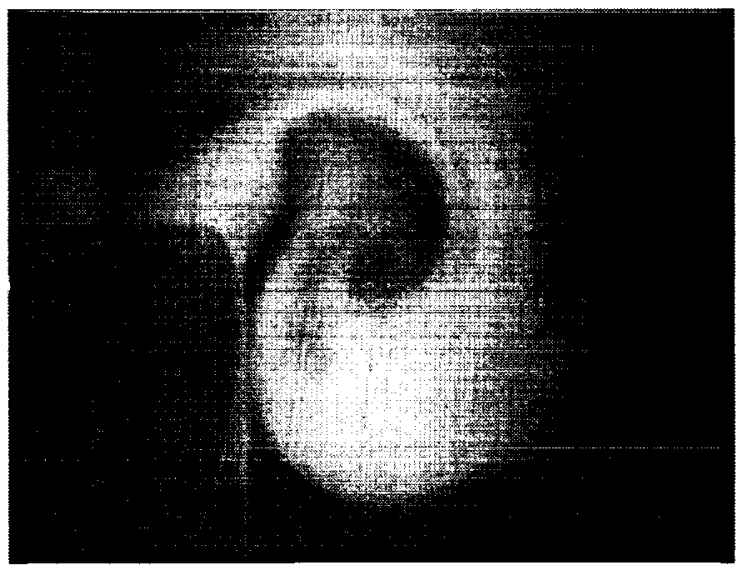

(b) 